\title{
1 Vortex Shedding Characteristics of Multi-Column Structure With or Without 2 Horizontal Connections
}

Department of Naval Architecture, Ocean and Marine Engineering, University of Strathclyde, Glasgow,

$5 \quad$ G4 OLZ, UK

\section{Abstract}

7 Vortex flow around multiple columns of finite length is ubiquitous in engineering. The present work 8 focuses on the basic fluid physics in terms of the vortex shedding flow patterns and their dependence on 9 structural configurations and flow parameters. Though widely documented in the literature, there is no consensus on certain aspects of the wake characteristics immediately behind the obstacles for a multicolumn structure at a relative high Reynolds number range. A comprehensive set of numerical simulations has been conducted to investigate the flow interactions with four square section shaped columns in a diamond configuration, which is complimented by experiments using particle image velocimetry and force measurements in a physical model with Reynolds numbers varying from $3.7 \times 10^{4}$ to $6.0 \times 10^{4}$. Horizontal structural members called pontoons were added near the end of the columns to alter the interactions with the surrounding fluid. This work reveals further insights of the fluid physics including the interactions of the vortex shedding processes due to the multi-columns and pontoons. The pontoons are seen blocking the vortices shed from the free end of the column by pushing the recirculation region further away from the free end of each column. In addition to the vortex shedding period being increased, further examination of the wake region indicates that the vortex street tends to be tidier and more structured by adding the pontoons to a basic multi-column structure. The findings will lead to better understanding in vortex shedding fluid physics and improved design in new offshore structure development such as deep-draft semi-submersibles and tension leg platforms.

\footnotetext{
*longbin.tao@strath.ac.uk
} 


\section{Nomenclature}

A Projected area of the immersed structure

$B_{L} \quad$ Overall width of the structure

$B_{T} \quad$ Draft of the structure

$C_{D} \quad$ Drag force coefficient

$C_{L} \quad$ Lift force coefficient

$C_{p} \quad$ Pressure coefficient

$D \quad$ Column projected width

$f \quad$ Vortex shedding frequency

$\mathrm{Fr} \quad$ Froude number

$F_{D} \quad$ Hydrodynamic drag force acting on the structure

$F_{L} \quad$ Hydrodynamic lift force acting on the structure

GCI Grid convergence index

$H_{1} \quad$ Immersed column height above the pontoon

$\mathrm{H}_{2} \quad$ Immersed column height

$L \quad$ Column width

$P \quad$ Pontoon height

Re Reynolds number

rms Root mean square

$S \quad$ Distance between centre columns

St Strouhal number

$\Delta t \quad$ Numerical simulation time step

$U \quad$ Current speed

$U_{i} \quad$ Streamwise flow velocity (velocity component $i$ )

$U_{j} \quad$ Transverse flow velocity (velocity component $j$ )

$U_{k} \quad$ Spanwise flow velocity (velocity component $k$ )

$\rho \quad$ Fresh water density

$\vec{\omega}_{x} \quad$ Streamwise vorticity

$\vec{\omega}_{y} \quad$ Transverse vorticity

$y^{+} \quad$ Y plus value

1. Introduction

Since Thom [1] accomplished an early study on the pressure distribution around a cylinder with low Reynolds numbers varying from 3.5 to 174 , flow around a cylinder has been a classical case for investigating the phenomena of flow separation. Norberg [2] summarized the results from several previous studies about the fluctuating forces on a circular cylinder. Recently, Parnaudeau et al. [3] investigated the flow over a circular cylinder both numerically with large eddy simulation and experimentally with hot-wire anemometry and particle image velocimetry (PIV) at Reynold number $=3900$. A significant amount of 
tandem arrangement by Bearman [4]. Although the case of the flow around a cylinder has been studied for many decades, study of a more complex arrangement such as four cylinders arrays is still rarely investigated and documented. The first investigation on the flow around four cylinders in a square arrangement was carried out by Sayers [5]. The force coefficients and vortex shedding frequencies were measured with different pitch ratios and flow incidences [5, 6]. Much of the research on the flow interactions among groups of four cylinders was done by Lam and co-authors over the last two decades [7-12]. The understanding of the mechanism on the multiple cylinder interaction is mainly based on their long-term investigation efforts. Lam and Lo [7] firstly performed experiments to investigate the flow patterns and the corresponding Strouhal number of four cylinders followed by an experiment in an open circuit wind tunnel [8]. In their work, the pressure distributions and force coefficients on each of the cylinders were measured to discuss the effects of incidence and spacing ratio. Following these studies, a water tunnel experiment at a Reynolds number 200 was carried out by Lam et al. [9]. The PIV technique was employed in their study to measure the velocity profiles as well as the flow patterns during the experiment. The force coefficients and vortex shedding frequencies were measured in a wind tunnel experiment by Lam et al. [10]. The downstream cylinders are always seen to be subjected to a higher lift force and lower drag forces than the upstream cylinders [10]. Lam et al. [11] used a numerical routine to simulate a cross-flow around four cylinders in an in-line arrangement and the spacing ratio effects were further studied by Lam and Zou [12] in more detail at a Reynolds number of 200. Other researchers, such as Zou et al. [13], Zhao and Cheng [14], and Wang et al. [15] also made their own contributions on the study of cross-flow around four cylinders. Especially, Wang et al. [15] carried out an experiment in an open water tunnel focusing on the vortex shedding patterns in the wake region of four cylinders. The cylinder spacing ratio and incidence angle effects on the vortex shedding patterns were illustrated. A good correlation between the flow pattern and the fluctuation forces was demonstrated in their study.

It is noted that the majority of studies on flow separation around cylinders are based on a simple infinite cylinder assumption. In most of engineering applications, however, structures comprised of multiple cylinders are often with finite length i.e., the existing of free end. It has attracted considerable attentions for the study of cross-flow past a cylinder with a free end, which is close to engineering applications such as poles, towers and offshore structures. Sumner [16] well documented the literature about flow around the cylinder with free end with a great deal of research work including 52 papers being summarized. Morton 

and Yarusevych [17] studied flow past a circular cylinder with a single stepwise discontinuity at two Reynolds numbers, 150 and 300. However, for the case of cross-flow around a group of cylinders each with free end, there is still a lack of understanding on this complex interaction. Liang et al. [18] conducted a preliminary study on flow past four free end rectangular section columns by both experimental and numerical routines. In this study, the cross-flow around four free end rectangular columns with and without pontoons in a diamond configuration, i.e. the flow incidence angle is at 45 degree, is investigated. Adding the pontoons at the base between the columns is found to significantly alter the flow patterns around the columns and lead to the change of flow parameters such as force coefficients and Strouhal number. The interaction between each column and the pontoons effects on the flow patterns are discussed.

\section{Experimental arrangement}

A series of experiments was conducted in the circulating water channel at Shanghai Jiao Tong University. The circulating water channel is vertically placed with an $8.0 \mathrm{~m}$ length, $3.0 \mathrm{~m}$ width and $1.6 \mathrm{~m}$ water depth measuring section. The current velocity range can be varied from $0.1 \mathrm{~m} / \mathrm{s}$ to $3.0 \mathrm{~m} / \mathrm{s}$, with the maximum allowable fluctuations of $0.01 \mathrm{~m} / \mathrm{s}$.

Table 1 Main characteristics of the four columns configuration (model I).

\begin{tabular}{lc}
\hline \hline & Model I \\
\hline Spacing ratio $(S / D)$ & 2.63 \\
Aspect ratio $\left(H_{1} / D\right)$ & 1.71 \\
Corner ratio $(R / D)$ & 0.11 \\
\hline \hline
\end{tabular}

Table 2 Main characteristics of the four columns with pontoons connected configuration (model II).

\begin{tabular}{lc}
\hline \hline & Model II \\
\hline Spacing ratio $(S / D)$ & 2.63 \\
Aspect ratio $\left(H_{2} / D\right)$ & 1.9 \\
Corner ratio $(R / D)$ & 0.11 \\
Pontoon height ratio $(P / D)$ & 0.36 \\
\hline \hline
\end{tabular}

Two sets of model test were conducted, including a four columns configuration (Model I) and a four columns with pontoons connected configuration (Model II). The projected width $(D)$ of the column is designed as $0.215 \mathrm{~m}$ in the present study. Details are presented in Table $\mathbf{1}$ and Table $\mathbf{2}$ as well as shown in Fig. 1. 


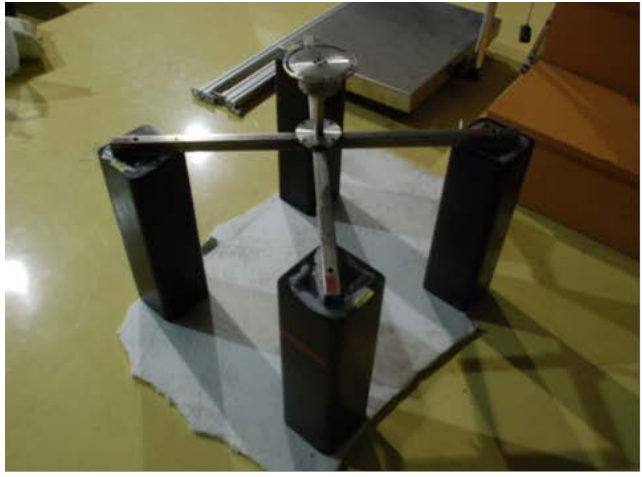

(A) Four columns configuration (model I).

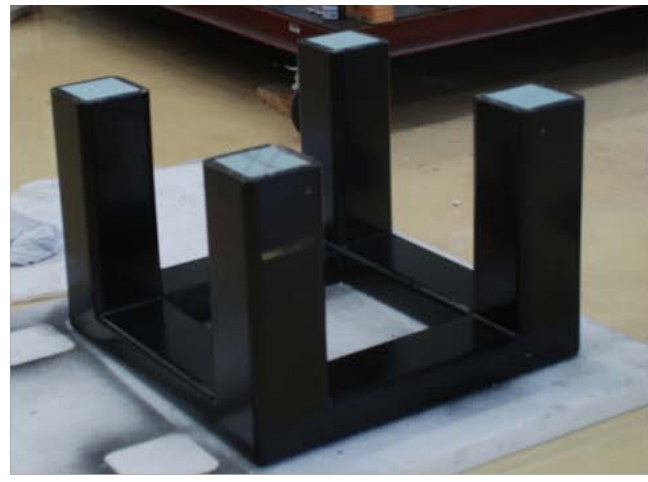

(B) Four columns with pontoons connected configuration (model II)

Fig. 1 Model configurations: (A) Four columns configuration (model I); (B) Four columns with pontoons connected configuration (model II).

The models were mounted to the cross-structure at the top of the test section and connected by a threecomponent force transducer capable of measuring the total fluid forces on the overall model. The central line of the model was coincided with the central line of the circulating water channel at in-line direction. For each individual test case, the experiments were run at least twice and the final results from the experiments are averaged from the two or more experimental data sets. With the aim to record images of flow patterns developing around and behind column 3 (see Fig. 2 for the definition of the column arrangement and numbering) during the experiments, the LaVision GmbH Particle imaging velocimetry (PIV) system (including a double pulsed Nd:YAG laser, with output energy of $425 \mathrm{~mJ} /$ pulse at $532 \mathrm{~nm}$ wavelength to illuminate the particles) was employed. A LaVision ImgaerProX11M CCD camera was used to record two-dimensional (2D) images of the illuminated field of view at a frequency of $4.52 \mathrm{~Hz}$. Two hundred images were collected for each individual case. To improve vector calculation in areas with the large velocity fluctuations in the wake region, calculations are performed using multiple interactions with decreasing interrogation window size $(128 \times 128$ to $48 \times 48 \mathrm{px})$, automatically adjusting window size and shape to local seeding density and flow gradients. The DaVis 8.2.2 package was further used to process the velocity and vorticity contours. The experimental set-up is shown in Fig. 3. In the present study, the models were tested under 45 degree incidence with Reynolds number $(R e=U D / v$, where $U$ is the free stream velocity, $D$ is the projected width of the column and $v$ is the kinematic viscosity of the fresh water) ranging from $3.7 \times 10^{4}$ to $6.0 \times 10^{4}$. 


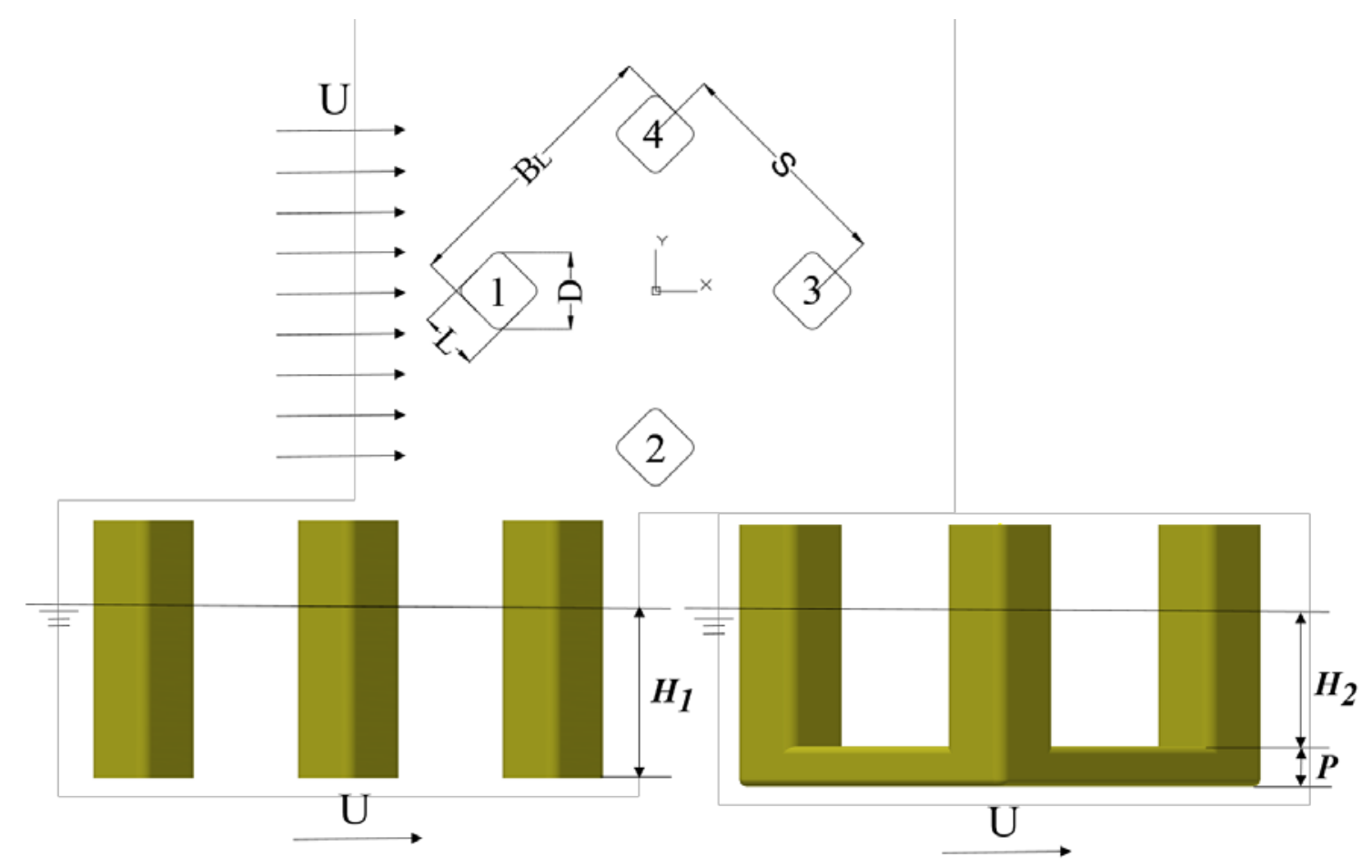

Fig. 2 Columns arrangement and flow direction.

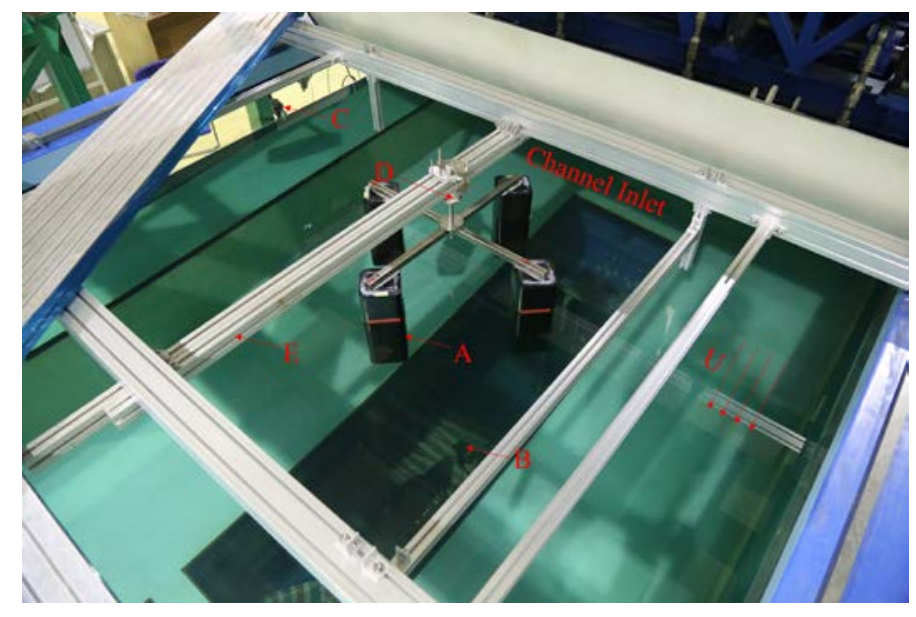
A) Model
B) PIV recording camera
C) PIV laser head
D) Three component force transducer
E) Adjustable support structure and top frame

Fig. 3 Experimental set-up in the circulating water channel.

\section{Numerical simulation}



$6.0 \times 10^{4}$. In the present numerical model, improved delayed detached eddy simulation (IDDES) [19] with Spalart-Almaras (SA) [20] was employed to improve the boundary layer simulation and in the meantime reduce the computational cost. The computational domain is divided into a number of non-uniform polyhedral grids [21] (see Fig. 4). For all simulations, a $9 B_{L} \times 6 B_{L} \times 3 B_{T}$ sized computational domain was used in the present study (where $B_{L}$ is the overall width of the structures and $B_{T}$ is the draft of the structure). Zou et al. [13] previously used a $32 L \times 20 L \times 3 L$ (about $7.1 B_{L} \times 4.4 B_{L} \times 3 L$ ) domain while the computational domains were $6 B_{L} \times 4.5 B_{L} \times 2.8 B_{T}$ and $5 B_{L} \times 4 B_{L} \times 2.2 B_{T}$ in the studies by Lee et al. [22]. Tan et al. [23] performed a numerical modelling using $27 B_{L} \times 18 B_{L} \times 6.5 B_{T}$ domain. Koop et al. [24], however, chose a $10 B_{L} \times 6 B_{T}$ cylindrical domain for simulating flow with their numerical models. In our earlier study [18], a computational domain size of $9.5 B_{L} \times 6.3 B_{L} \times 3 B_{T}$ was employed. Compared with aforementioned computational domain sizes, a $9 B_{L} \times 6 B_{L} \times 3 B_{T}$ domain was considered to be sufficiently cross flow direction.

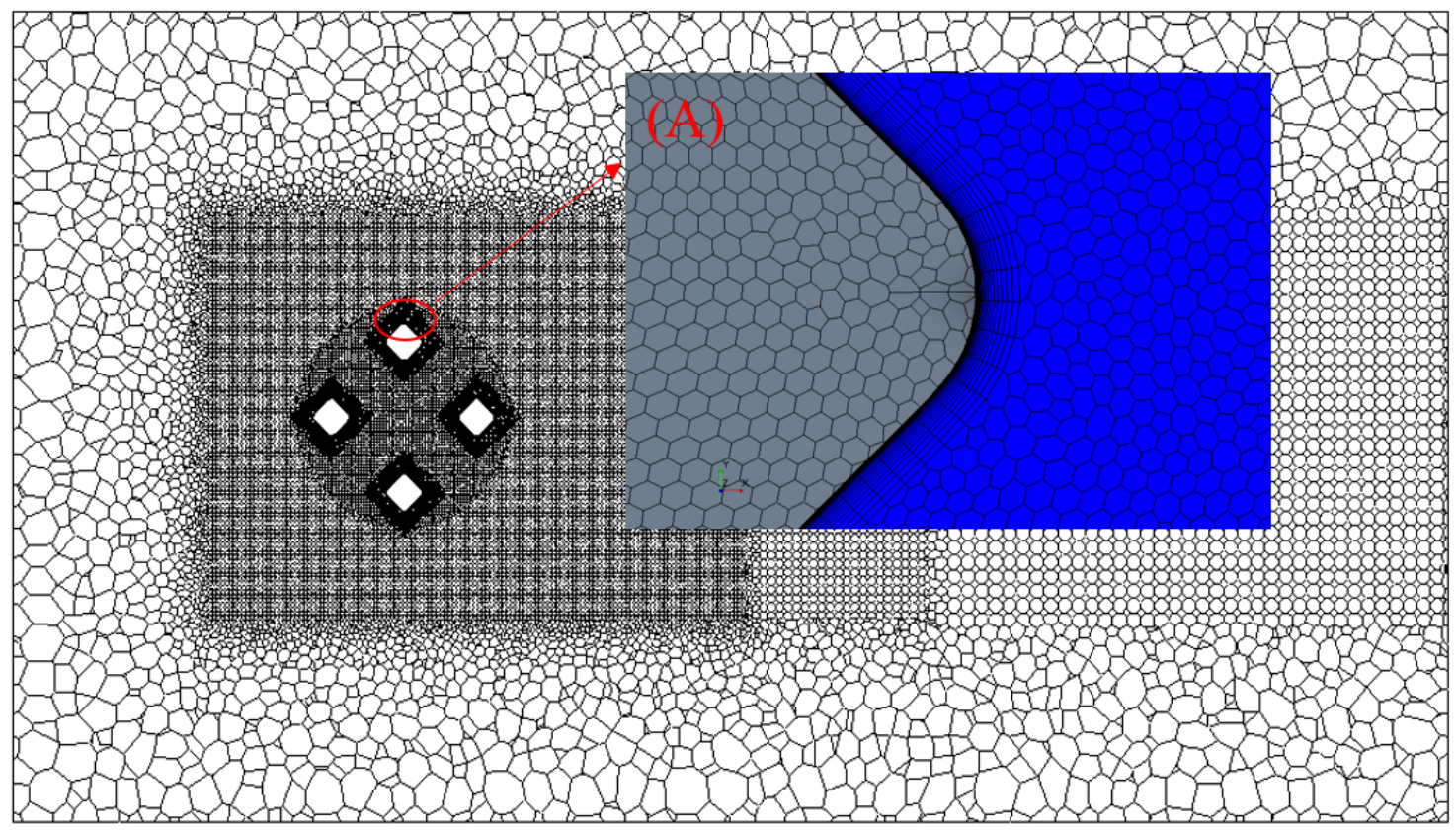

Fig. 4 Visualization of the mesh (Case: FCP_N5) at the middle draft level of the four columns 

method (FVM) is adopted to discretize the incompressible flow field equations [25]. The second-order implicit three time levels (ITTL) scheme is applied for the temporal discretization. The convective term is evaluated by using a hybrid second-order upwind scheme. SIMPLE algorithm is employed to treat the pressure and velocity coupling.

The computational domain was modelled with a three-dimensional mesh of elements. A polyhedral mesh [21] was used in this study. The overall element mesh domain is illustrated at a mid-depth horizontal layer in Fig. 4. In the present study, a near wall refinement method named "Prism Layer Mesher [21]" was adopted. The "Prism Layer Mesher" model (as shown in Fig. 4) is used with a core volume mesh to generate orthogonal prismatic cells next to wall surfaces. This layer of cells is necessary to improve the accuracy of the flow solution [21].

The boundary conditions for the present numerical model are summarised as follows: region is resolved with the large eddy simulation (LES). The IDDES simulations produced two logarithmic than the distance to the wall [19]. With the development from Shur et al. [19], a new hybrid model, IDDES is shown capable of resolving the issue of mismatch between the modelled log layer and the resolved log layer, which has been typical in either DES or DDES use for WMLES. This, however, requires rather strict Courant numbers, which is referred to a suitable time step setting. In the present study, the non-dimensional width of the column) is set as 0.008 to ensure the Courant number less than 1 (only in some tiny flow areas 
closed to the wall, the Courant number may reach to 1.5). Based on the recent study of modelling vortexinduced motions of a deep-draft semi-submersible [26, 27], the selected time step is considered to be fine enough for the current simulations’ requirement.

Additionally, the IDDES with SA approach can improve the performance of the boundary layer simulation under a strict $y^{+}$values requirement $\left(y^{+} \leq 1, y^{+}=u_{*} \Delta y_{1} / v\right.$, where $u_{*}$ denotes the friction velocity at the nearest wall, $\Delta y_{1}$ is the first layer thickness and $v$ is the kinematic viscosity). In the present study, the distance from the column surface to the nearest grids is specified to ensure the $y^{+}$values be smaller than 1 for all the simulations.

\subsection{Sensitivity study}

To verify reliability and accuracy of the numerical model, a mesh sensitivity study was carried out with different levels of grid refinement on each model. The convergence lines illustrate results for the physical quantities and non-dimensional flow parameter including the mean drag force coefficient $\left(\bar{C}_{D}\right)$, the root mean square lift force coefficient $\left(C_{\mathrm{Lrms}}\right)$, and the Strouhal number $(S t)$, which are defined as:

$$
\begin{aligned}
& C_{D}=\frac{F_{D}}{\frac{1}{2} \rho U^{2} A}, \\
& C_{L}=\frac{F_{L}}{\frac{1}{2} \rho U^{2} A}, \\
& S t=\frac{f L}{U},
\end{aligned}
$$

where, $F_{D}$ is the drag force on the structure, $F_{L}$ is the lift force on the structure, $\rho$ is the fresh water density, $U$ is the free stream velocity, $A$ is the projected area of the immersed structure. In Eq. (3), $f$ is the vortex shedding frequency obtained from the power spectra of lift force coefficient fluctuations as suggested by Schewe [28] and $L$ is the width of the column.

\subsubsection{Four columns configuration}

Table 3 Mesh sensitivity study for the four columns configuration.

\begin{tabular}{llccl}
\hline \hline Case & Elements (million) & $\bar{C}_{D}$ & $C_{\text {Lrms }}$ & $S t$ \\
\hline FC_N1 & 0.02 & 0.931 & 0.105 & 0.127 \\
FC_N2 & 0.13 & 1.052 & 0.111 & 0.139 \\
FC_N3 & 0.95 & 1.065 & 0.078 & 0.139 \\
FC_N4 & 3.50 & 1.068 & 0.066 & 0.150 \\
FC_N5 & 8.18 & 1.100 & 0.065 & 0.145 \\
\hline \hline
\end{tabular}


213 to a relatively fine grid refinement. The details of the sensitivity study and the corresponding simulation 214 results are shown in Table 3. The results are obtained by averaging over more than twenty vortex shedding cycles of the whole structure. The convergences are further illustrated in Fig. 5, Fig. 6 and Fig. 7.

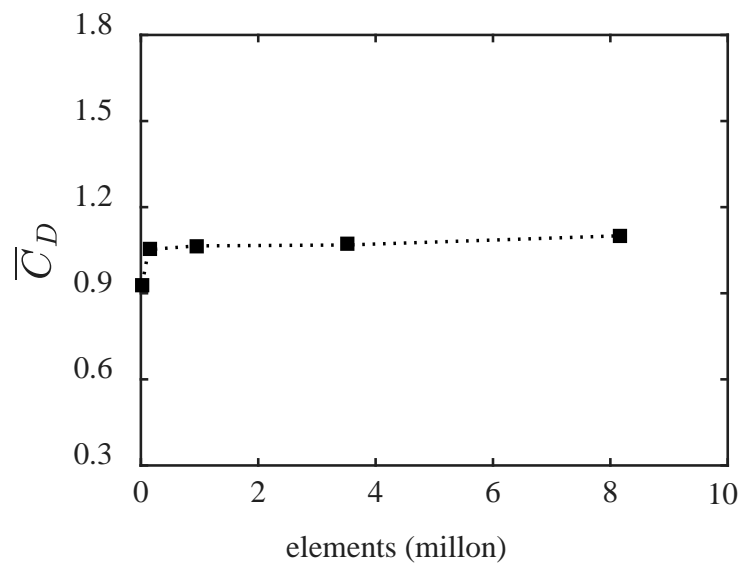

217 Fig. 5 Convergence of mean drag force coefficient $\left(\bar{C}_{D}\right)$ for the four columns configuration.

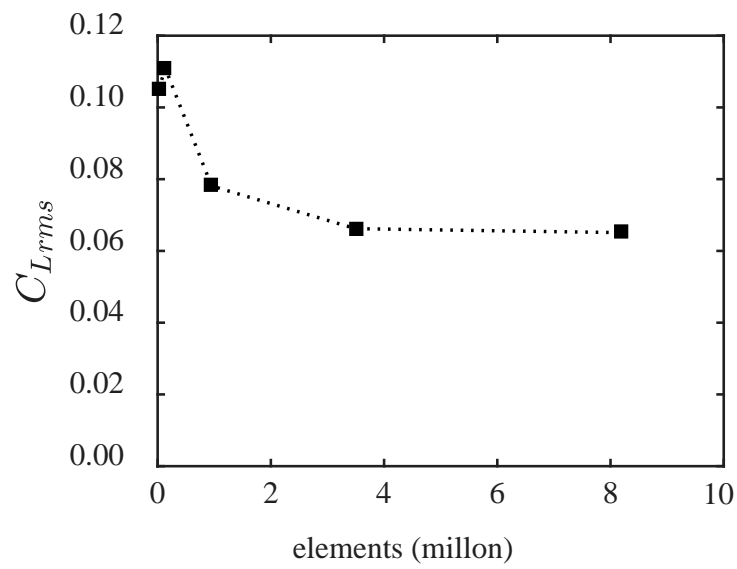

Fig. 6 Convergence of root mean square lift force coefficient $\left(C_{L r m s}\right)$ for the four columns configuration. 


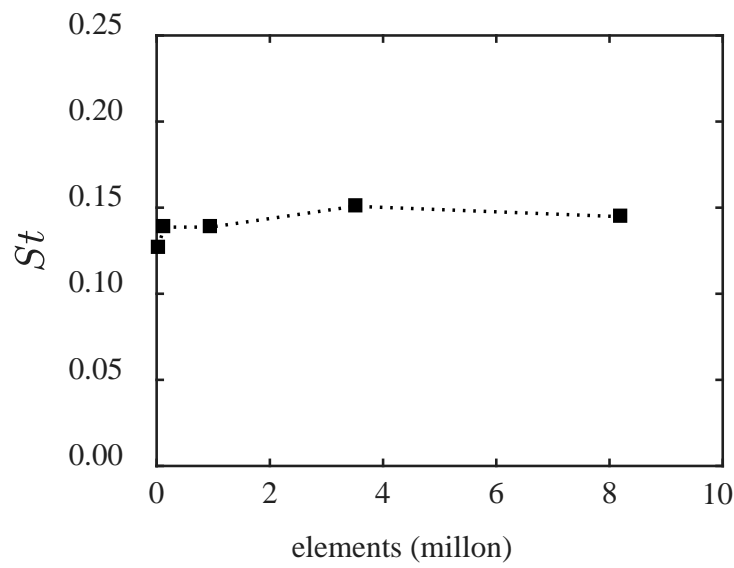

Fig. 7 Convergence of Strouhal number (St) for the four columns configuration.

As shown in Fig. 5, Fig. 6 and Fig. 7, the mesh refinement setting FC_N4 is considered to be fine enough and has been chosen for further numerical simulations and comparisons against the experimental data.

\subsubsection{Four columns with horizontal pontoons connected configuration}

Table 4 Mesh sensitivity study for the four columns with pontoons connected configuration.

\begin{tabular}{llccl}
\hline \hline Case & Elements (million) & $\bar{C}_{D}$ & $C_{\text {Lrms }}$ & St \\
\hline FCP_N1 & 0.15 & 1.030 & 0.171 & 0.122 \\
FCP_N2 & 0.56 & 1.064 & 0.178 & 0.122 \\
FCP_N3 & 0.94 & 1.053 & 0.139 & 0.134 \\
FCP_N4 & 3.43 & 1.068 & 0.101 & 0.131 \\
FCP_N5 & 6.86 & 1.066 & 0.093 & 0.131 \\
\hline \hline
\end{tabular}

227 As the configuration of the model is changed with the addition of the horizontal structure member pontoons, the mesh sensitivity study needs to be re-conducted. With the same methods being described in the above section for the four columns configuration, the convergence (see Fig. 8, Fig. 9 and Fig. 10) is performed with the results given in Table 4 . 


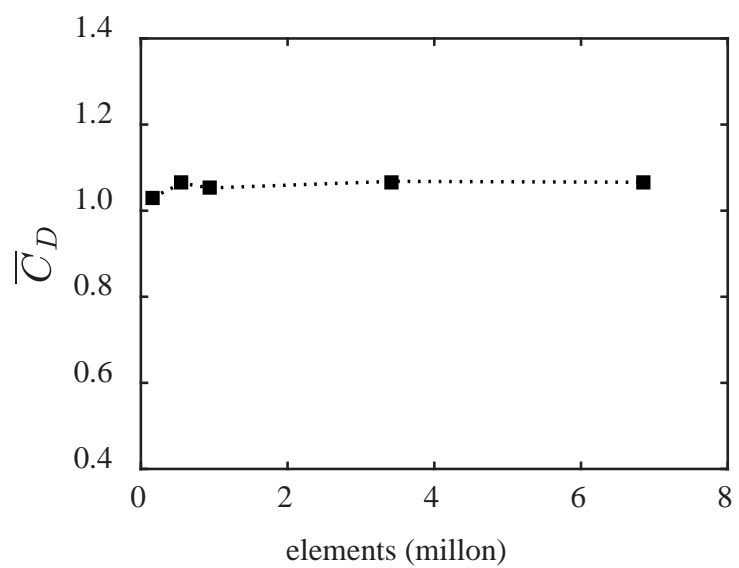

231

Fig. 8 Convergence of mean drag force coefficient $\left(\bar{C}_{D}\right)$ for the four columns with pontoons connected 233 configuration.

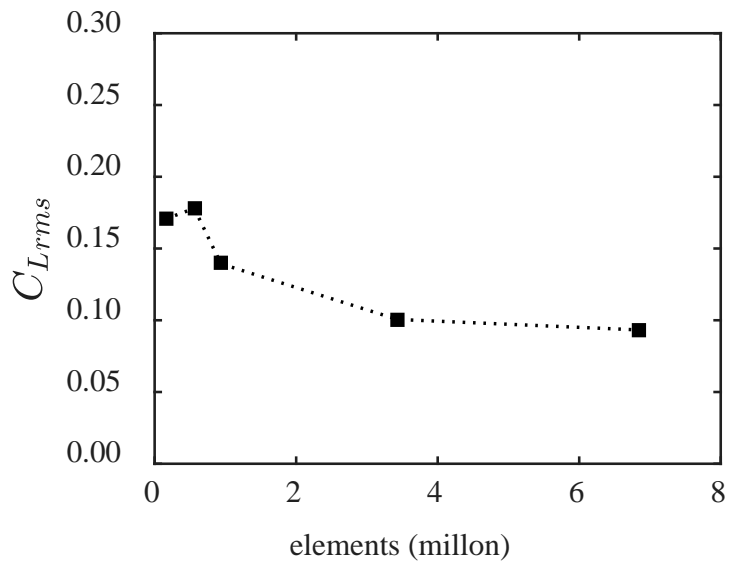

234

Fig. 9 Convergence of root mean square lift force coefficient $\left(\mathrm{C}_{\mathrm{Lrms}}\right)$ for the four columns with pontoons connected configuration.

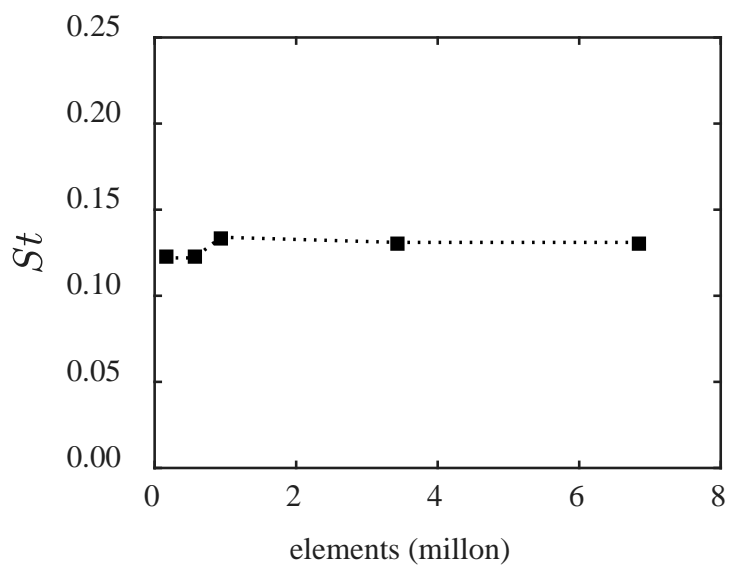



configuration.

The detailed mesh sensitivity study shows that the mesh setting FCP_N4 is fine enough to obtain confident results within an acceptable computation time. Thus, it is used in further numerical simulations for the four columns with pontoons configuration.

Additionally, based on the recent study of modelling vortex-induced motions of a deep-draft semisubmersible [26, 27], the selected mesh is considered to be fine enough for the current simulations' requirement.

\section{Results and analyses}

247 Two different configurations (columns with and without pontoons connected) under 45 degree flow incidence were investigated using the established numerical models and their results are further compared against the corresponding experimental data.

250

251

252

253

254

\subsection{Flow characteristics in the horizontal plane}

In order to reveal further insights of the fluid physics due to the fluid flow interaction with multiple columns and pontoons arrangements, a general visual appreciation of the vortex shedding patterns (e.g. the time-averaged velocity contours and the vorticity contours) at $R e=4.3 \times 10^{4}$ from both the experimental measurements and the numerical simulations are presented and the flow characteristics are analysed in this section.

\subsubsection{Time-averaged velocity distribution}

The velocity profiles in the wake region around column 3 in a horizontal $X Y$ plane (at the middle draft) obtained from the experiments and numerical simulations for the two configurations are shown in Fig. 11 and Fig. 12 respectively (where $U_{\text {mean }}=\sqrt{\bar{U}_{l}^{2}+\bar{U}_{J}^{2}}, U_{i}$ is the streamwise flow velocity, $U_{j}$ is the transverse flow velocity). The velocities were sampled over the horizontal cross section coinciding with $X Y$ plane ( $i$, $j$ plane) to have direct quantifiable comparisons between the experimental PIV measurements and the numerical predictions (shown in Fig. 13 and Fig. 14). A typical characteristic U-shaped velocity profile is observed in Fig. 14. The numerical results show a good agreement with the experimental data, especially in capturing the recirculation flows in the wake region. The numerical simulations give similar velocity 
values to those from the experimental measurements. As one of the highlights in the present experiments, the PIV measurements have collected the flow data from an extended area (about $0.6 \mathrm{~m}^{2}$ measurement area) compared with the studies of previous researchers $[15,29]$, which is crucial to provide a more complete picture of the flow characteristics of the wider region around the columns. There are larger discrepancies observed at the sides of column 3 which is in the areas close to the edge of the camera screen. This is partly due to the resolution of the PIV image in these regions being often not as good as in the central part of the camera lens. The numerical predictions agree well compared with the experimental results at the central part of the lens (around $y / D=0$ ). As the present numerical model has been rigorously validated through both kinematics and dynamics, the relatively large discrepancies at the side are attributed to the poor resolution of the PIV image in the experiments. In general, the numerical simulations predict well compared with the experimental results.

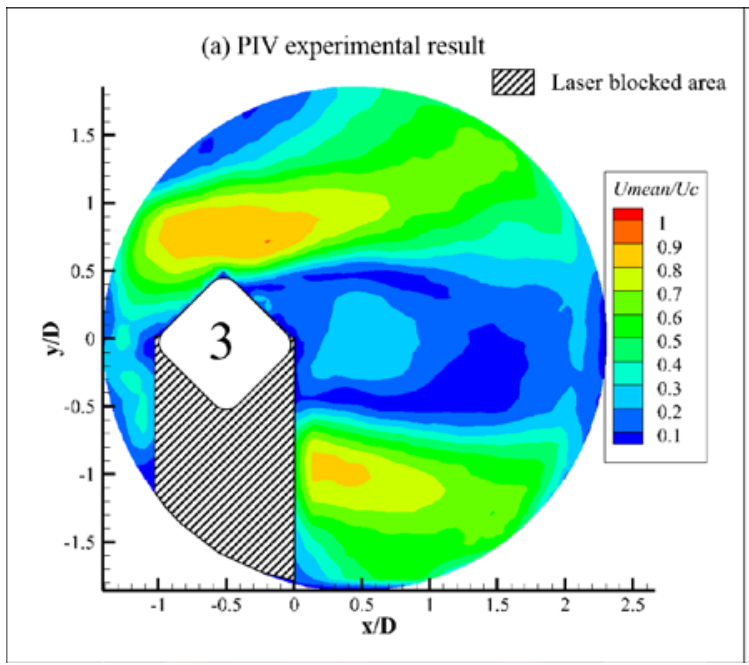

(b) Numerical result

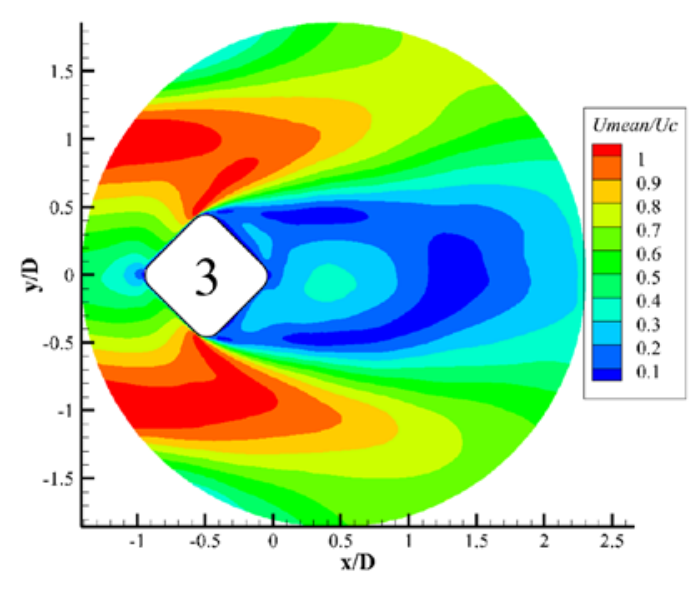

Fig. 11 Time-averaged velocity distribution behind column 3 at the middle draft level of the four columns configuration in $X Y$ plane. 

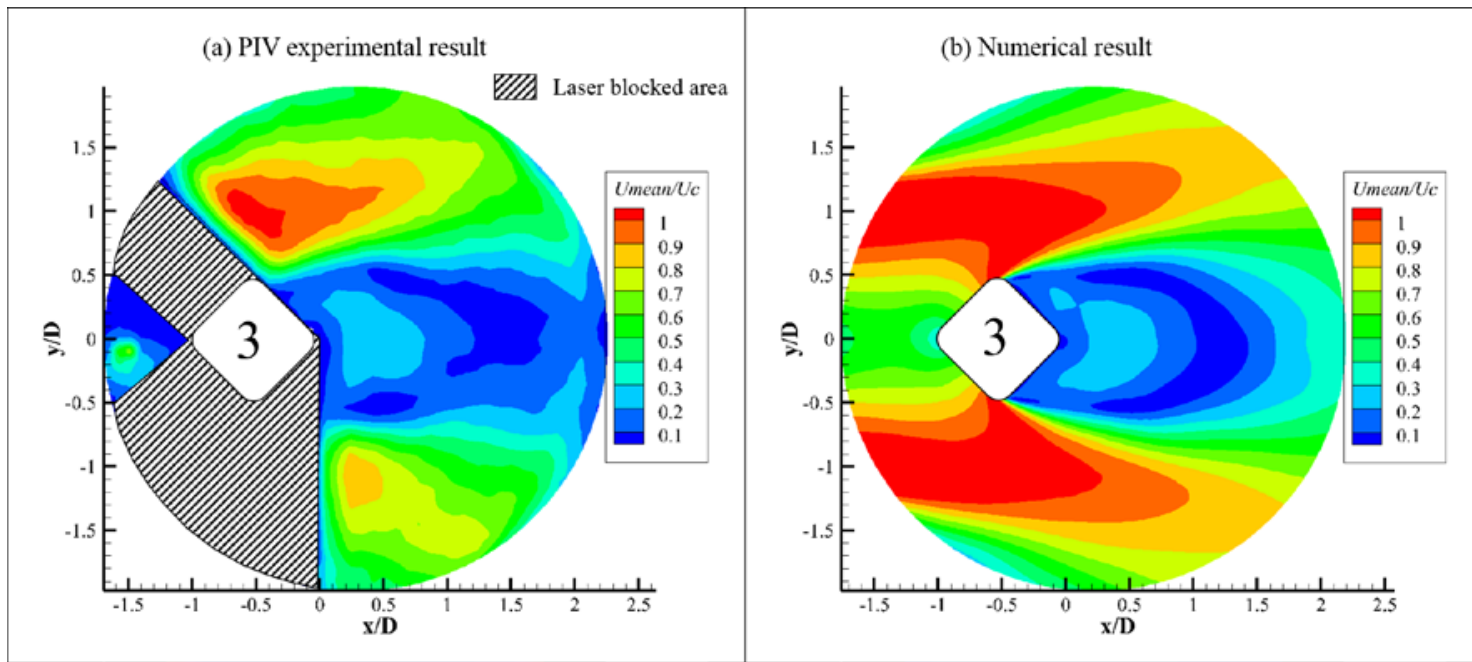

Fig. 12 Time-averaged velocity distribution behind column 3 at the middle draft level of the four columns with pontoons connected configuration in $X Y$ plane.
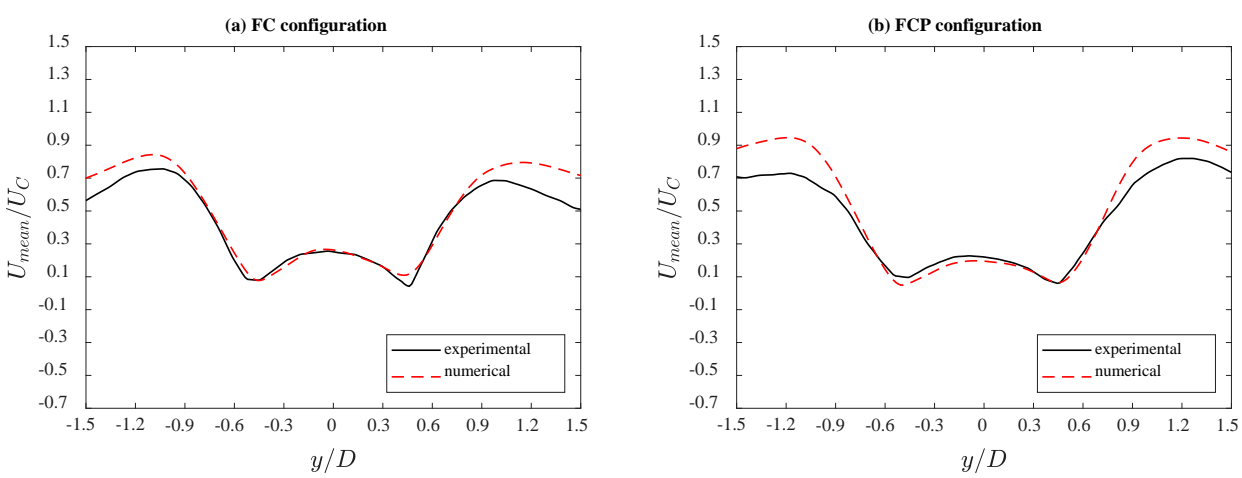

Fig. 13 Time-averaged flow properties of the $U_{\text {mean }} / U_{C}$ in $X Y$ plane (middle draft level of the structure) for column 3, at $x / D=0.75 R e=4.3 \times 10^{4}$, "FC" is the four columns configuration and "FCP" is the four columns with pontoons connected configuration.
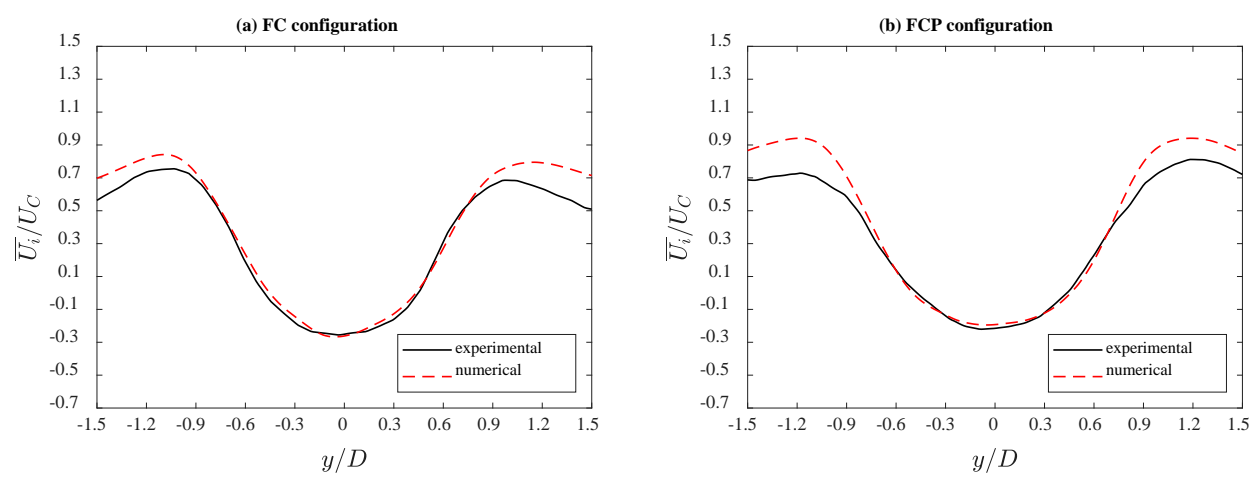
Fig. 14 Time-averaged flow properties of the $\overline{U_{i}} / U_{C}$ (velocity component $i$ ) in $X Y$ plane (middle draft level of the structure) for column 3 at $x / D=0.75, R e=4.3 \times 10^{4}$. "FC" is the four columns configuration and "FCP" is the four columns with pontoons connected configuration.

It is noted that the experimental data are quite limited due to a limitation of the PIV measuring range. Detailed examination of the flow characteristics around the complete structural configuration is based on 292 the comprehensive numerical results in the present work. The time-averaged streamwise velocity components (velocity component $i$ ) downstream of each column are shown in Fig. 15. By adding the

294 pontoons into the structure, the minimum values of the streamwise velocities after each column are seen to be slightly increased to different levels. For column 1, it is seen that the pontoons increases streamwise velocity in the recirculating region, but it decreases the velocity distribution trend at the sides of the column. Unlike the downstream flow of column 1, the downstream flow of column 3 shows a different trend of the velocity at the two sides of the column, a clear indication of the upstream turbulence effect to the downstream velocity profile. Fig. 15 also shows a larger streamwise velocity around the four columns with pontoons connected configuration than that around the four columns configuration. At the recirculation region, the velocity trend turns out to be flatter for the four columns with pontoons connected configuration. For the two side columns (column 2 and 4), as can be seen in Fig. 15, their streamwise velocity distributions are symmetric with respect to the centre line of the whole structure. 

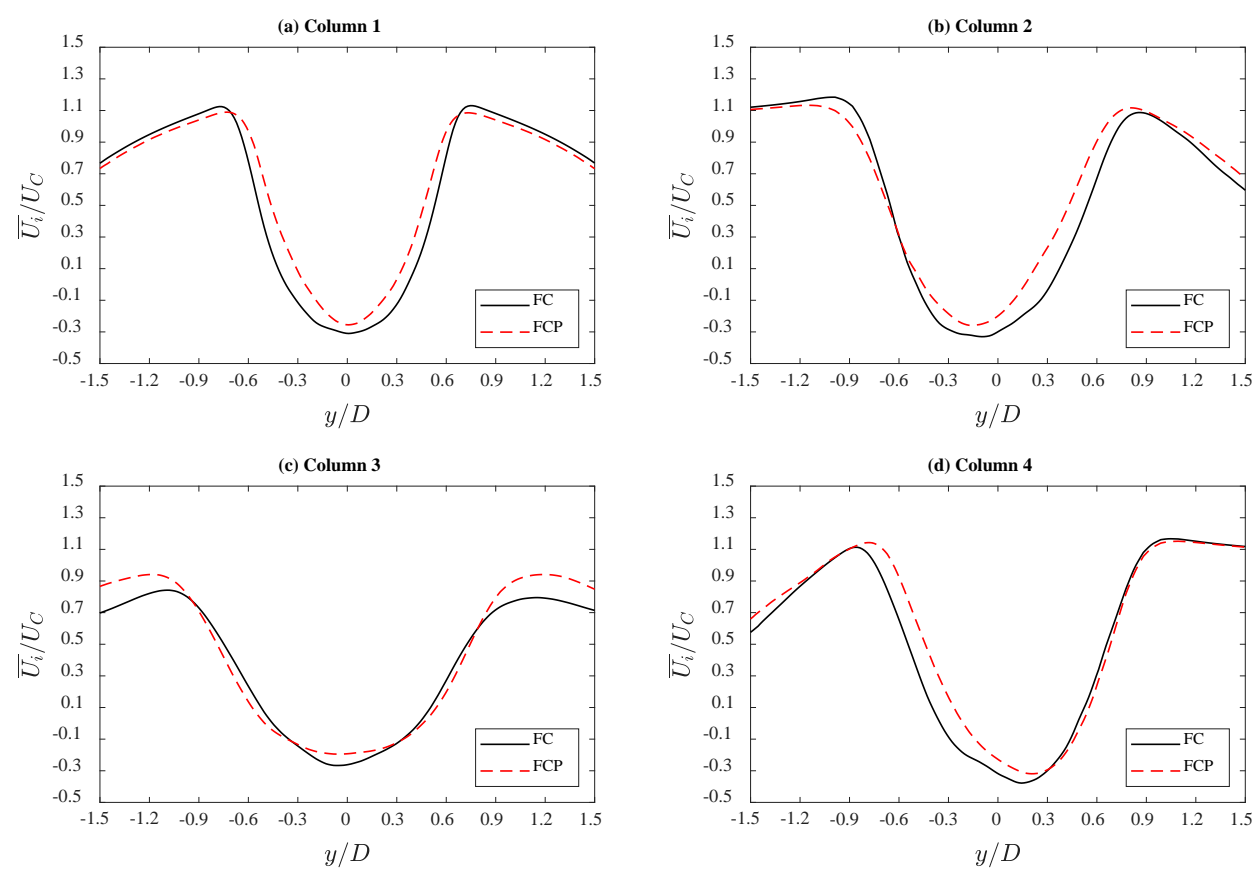

Fig. 15 Numerical predicted time-averaged flow properties of the $\overline{U_{i}} / U_{C}$ (velocity component $i$ ) in $X Y$ plane (middle draft level of the structure) for each column at $x / D=0.75, R e=4.3 \times 10^{4}$. "FC" is the four columns configuration and "FCP" is the four columns with pontoons connected configuration.

\subsubsection{Instantaneous vorticity contour and wake forms}

The instantaneous vorticity contours have always been closely related to the fluid physics, especially in the vortex flow. In the present section, the instantaneous non-dimensional vorticity $\left(\vec{\omega}_{z} D / U\right)$ contours obtained from the experiments within one vortex shedding period around column 3 for both configurations are shown in Fig. 16 and Fig. 17 respectively. These vorticity contours demonstrated the wake detailing the vortex formation and shedding. It is noted that the comparison of the flow characteristics between the two configurations is made for the four time instances equally spaced within one vortex shedding period rather than the exact fixed time instances. As can be seen in these figures, due to the effects of the vortices shed from the three upstream columns, the wake region behind column 3 is full of fragmentized vortices. The vortices shed from column 3 quickly break into pieces and mix with the vortices shed from the two side columns (column 2 and 4) after they have been separated from the column by the ambient flow. It is also noticed that, by adding the pontoons, the vortex street trailing from column 3 becomes clearer and that the vortices shed from column 3 break up relatively slowly compared with the four columns configuration. 
321 Strong vortices can be found flowing afterward from column 3 in the four columns with pontoons connected configuration, indicating a clearer wake forming process observed by adding the pontoons.
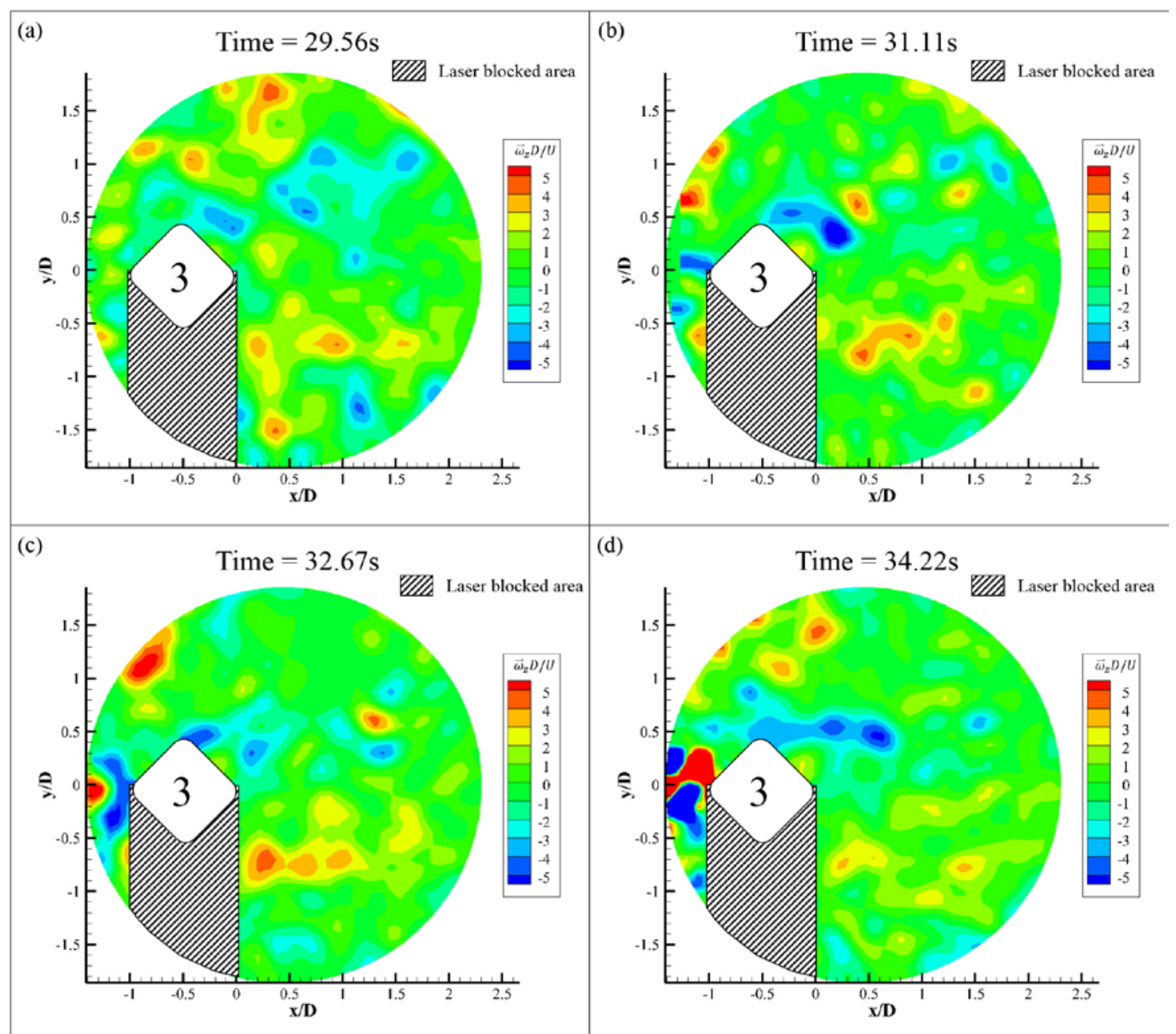

Fig. 16 Instantaneous flow fields around column 3 for the four columns configuration at $X Y$ plane (middle draft level of the structure), where $\vec{\omega}_{z} D / U$ is the non-dimensional spanwise vorticity. 

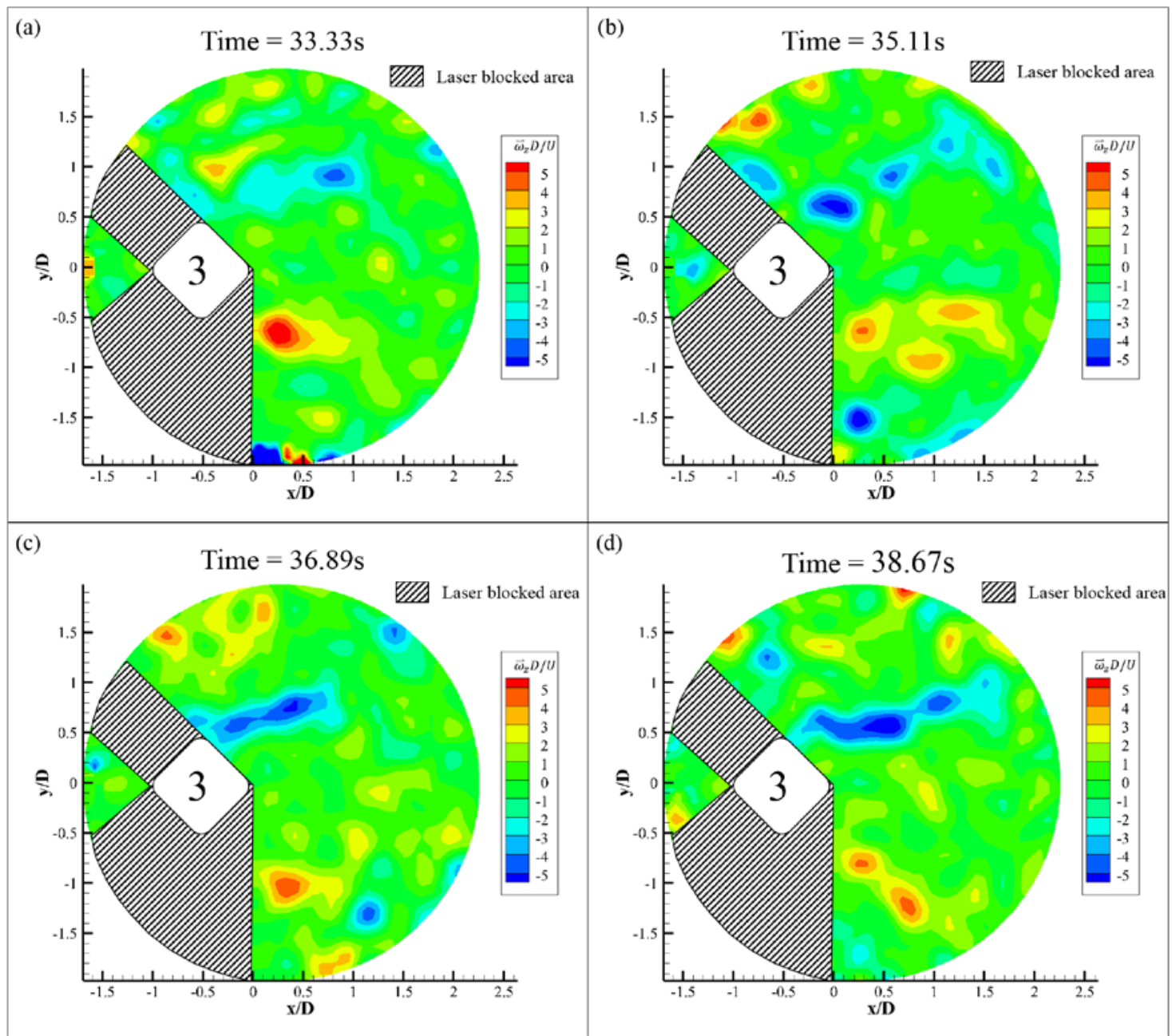

Fig. 17 Instantaneous flow fields around column 3 for the four columns with pontoons connected configuration at $X Y$ plane (middle draft level of the structure), where $\vec{\omega}_{z} D / U$ is the non-dimensional spanwise vorticity.

Table 5 The chronological order of vortices genesis for each column.

\begin{tabular}{|c|c|c|}
\hline Column & Shear layer & Vortex street \\
\hline \multirow{2}{*}{1} & Upper & $\mathrm{A} 1, \mathrm{~A} 2 \ldots$ \\
\hline & Lower & B1, B2 ... \\
\hline \multirow{2}{*}{2} & Upper & $\mathrm{C} 1, \mathrm{C} 2 \ldots$ \\
\hline & Lower & $\mathrm{D} 1, \mathrm{D} 2 \ldots$ \\
\hline \multirow{2}{*}{3} & Upper & E1, E2 ... \\
\hline & Lower & F1, F2 ... \\
\hline \multirow{2}{*}{4} & Upper & G1, G2 ... \\
\hline & Lower & $\mathrm{H} 1, \mathrm{H} 2 \ldots$ \\
\hline
\end{tabular}



numerical simulations for the two different configurations are shown in Fig. 18 and Fig. 19. For convenience in describing the vortex development processes, the vortices shed from each side of the column are denoted in chronological order of genesis (e.g., A1, A2 ... from the upper side of column 1, see Table 5). It is noted that the description of vortex shedding development is intended to reveal the formation and development of the wake.

As shown in Fig. 18, for the four columns configuration, the vortices shed from each side of the individual column break into pieces immediately after being separated from the columns. There are a large number of small vortices near the reverse side of the columns in the downstream weak area, especially for column 1. The vortices shed from the upstream column (column 1) are seen directly impinging on the downstream column (column 3), and subsequently (see B1 in Fig. 18(c)) break into two parts and then joined into the wake region of the other three columns (see two B1' in Fig. 18(d)). Additionally, the vortices shed from the inner sides of the portside and the starboard side columns (column 2 and 4) break into pieces rapidly after shedding from the inner corner of the respective columns (see C1, H1 in Fig. 18). However, the vortices shed from the outer sides of the portside and the starboard side columns appear to remain for a relatively long time compared to their counterparts shed from inner side corners (see D1, D2, G1, G2 in

347 Fig. 18). It is evident however, vortex shedding formation of the downstream column 3 shows a rather different phenomenon. The vortices (see E1, F1 in Fig. 18) shed from column 3 appear to remain in one position for a certain duration and subsequently break into two parts. It is noted that only a portion of the vortices join into the wake region of the whole structure with the remaining part of the vortices impinging reversely on column 3 (see two F1' in Fig. 18(d)). The whole flow region around the four columns shown in Fig. 16). 

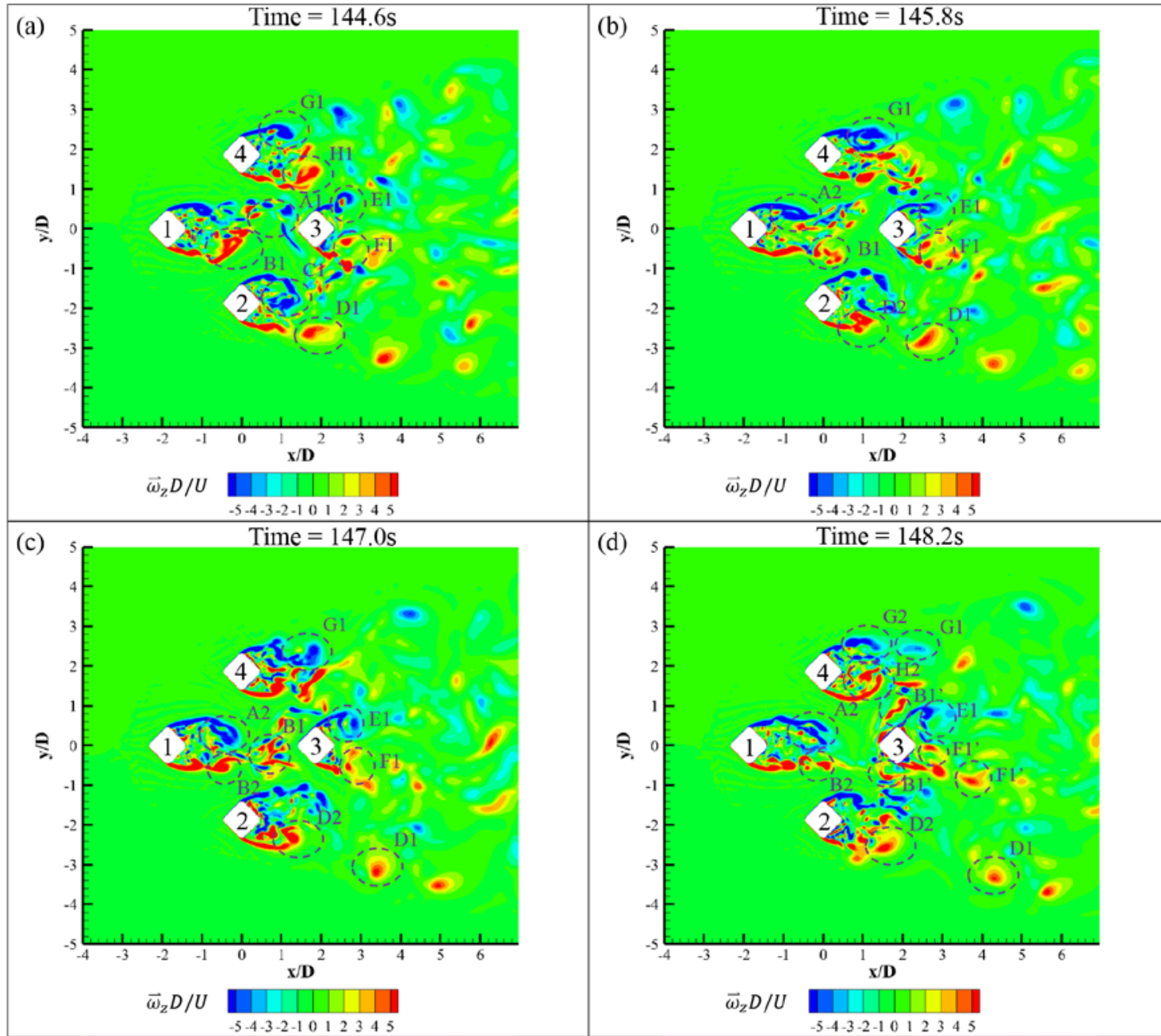

Fig. 18 A time series of the non-dimensional spanwise vorticity $\left(\vec{\omega}_{z} D / U\right)$ contours around the four columns configuration at the middle draft level showing the instantaneous flow fields in $X Y$ plane at $R e=4.3 \times 10^{4}$.

By adding the pontoons to the structure, the flow characteristics were appreciably altered. As shown in

Fig. 19, compared with the four columns configuration, the striking feature is that the vortices shed from each column become more structured and clearly defined. Very slim vortices are shed from the corners of column 1 and subsequently impinge on column 3. Strong vortices are observed from both the portside and starboard side columns (column 2 and 4), in particular from the outer corners of the two side columns. It is observed that the vortices (see H1 and C2 in Fig. 19) shed from the inner sides of the side columns break into two pieces (see H1' and C2' in Fig. 19). Unlike the four columns configuration, with the pontoons connecting between the columns, the vortices shed from the downstream column (column 3) are seen to quickly disappear after they separate from the corner of column 3 (see E1, E2, F1 and F2 in Fig. 19). Thus, 

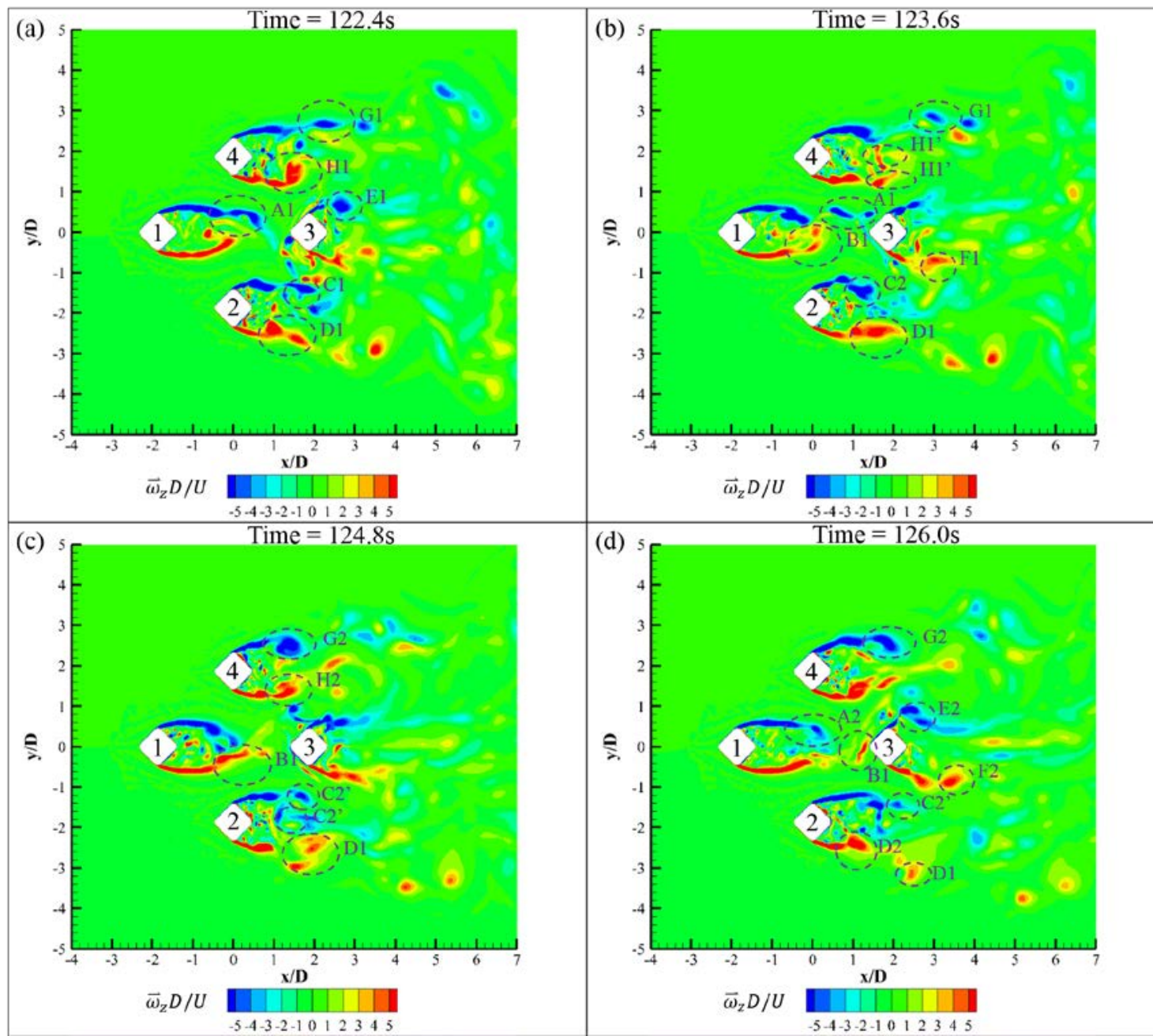

Fig. 19 A time series of the non-dimensional spanwise vorticity $\left(\vec{\omega}_{z} D / U\right)$ contours around the four columns with pontoons connected configuration at the middle draft level showing the instantaneous flow fields in $X Y$ plane at $R e=4.3 \times 10^{4}$.

\subsubsection{Flow separation patterns}

To further examine the flow separation patterns, the streamlines along with the vorticity contour are illustrated in Fig. 20 to Fig. 23.

As can be observed in Fig. 20 and Fig. 21, the number of circulating region closed to column 4 is decreased by adding the pontoons. This further indicates that adding the pontoons to the structure resulting more stable flow separation pattern by limiting the end effect of the columns compared to the four columns 
only configuration. Additionally, with the observations at different draft levels of the structure, it shows that the width of the wake region is decreased by adding the pontoons to the structure. Unlike the four columns only structure, with the pontoons connecting the columns, the flow separation patterns change significantly. It can be seen in Fig. 21, the flow separation is extended markedly further downstream when the draft level is close to the pontoons. However, for the four columns only configuration, the flow separation is quite similar in Fig. 20.
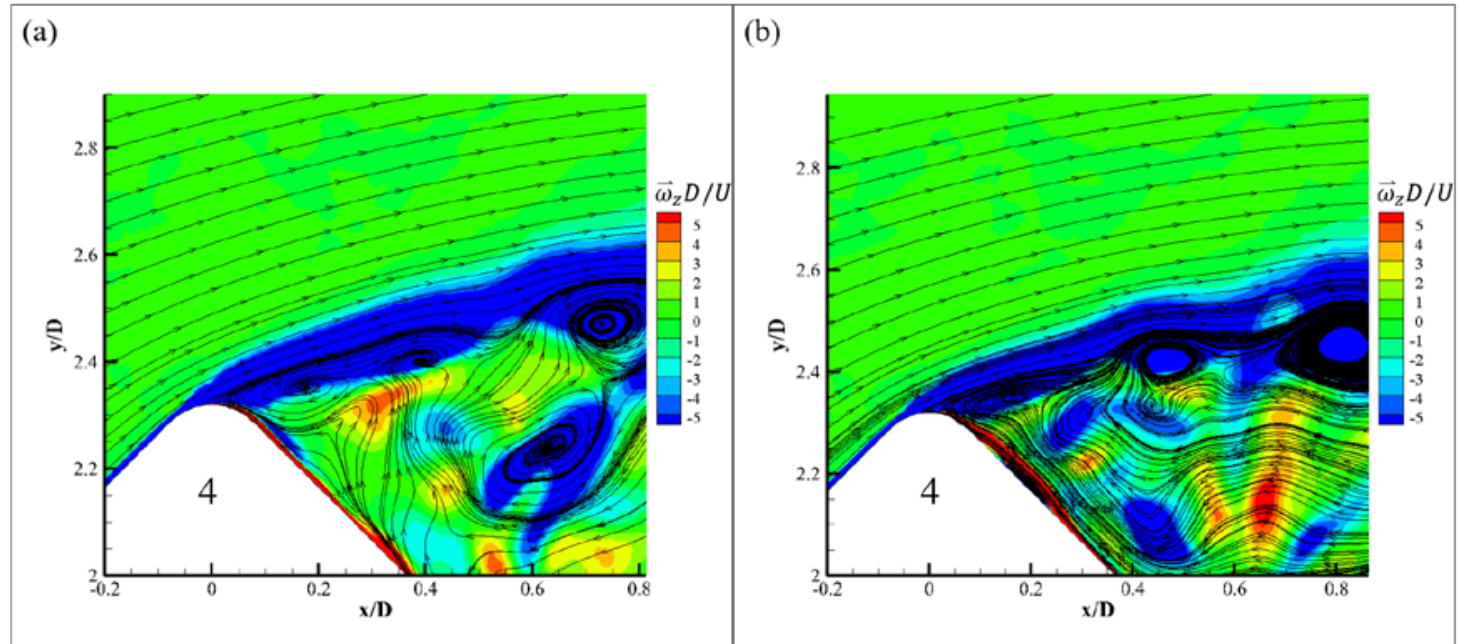

(c)

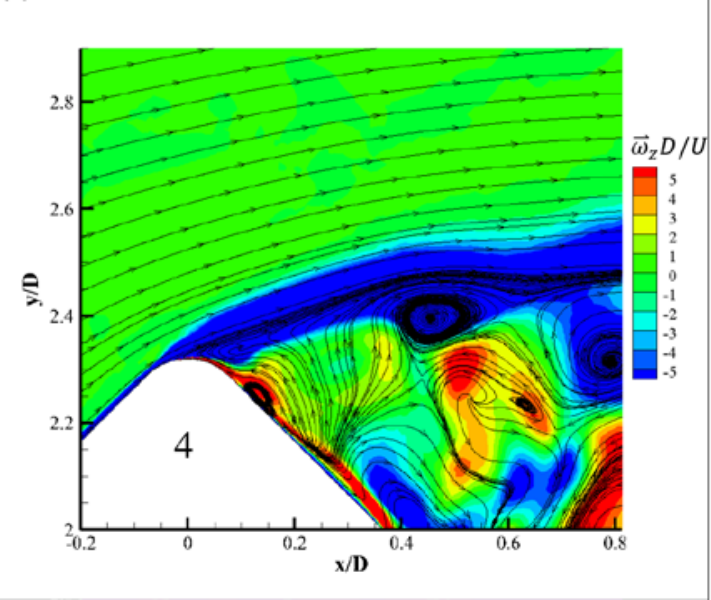

Fig. 20 The streamlines along with the vorticity $\left(\vec{\omega}_{z} D / U\right)$ contours around column 4 (the four columns configuration) at different draft levels (a: middle draft level; b: $z=0.13075 \mathrm{~m}$ draft level; c: pontoon upper face draft level) showing the flow separation in $X Y$ plane at $R e=4.3 \times 10^{4}$. 


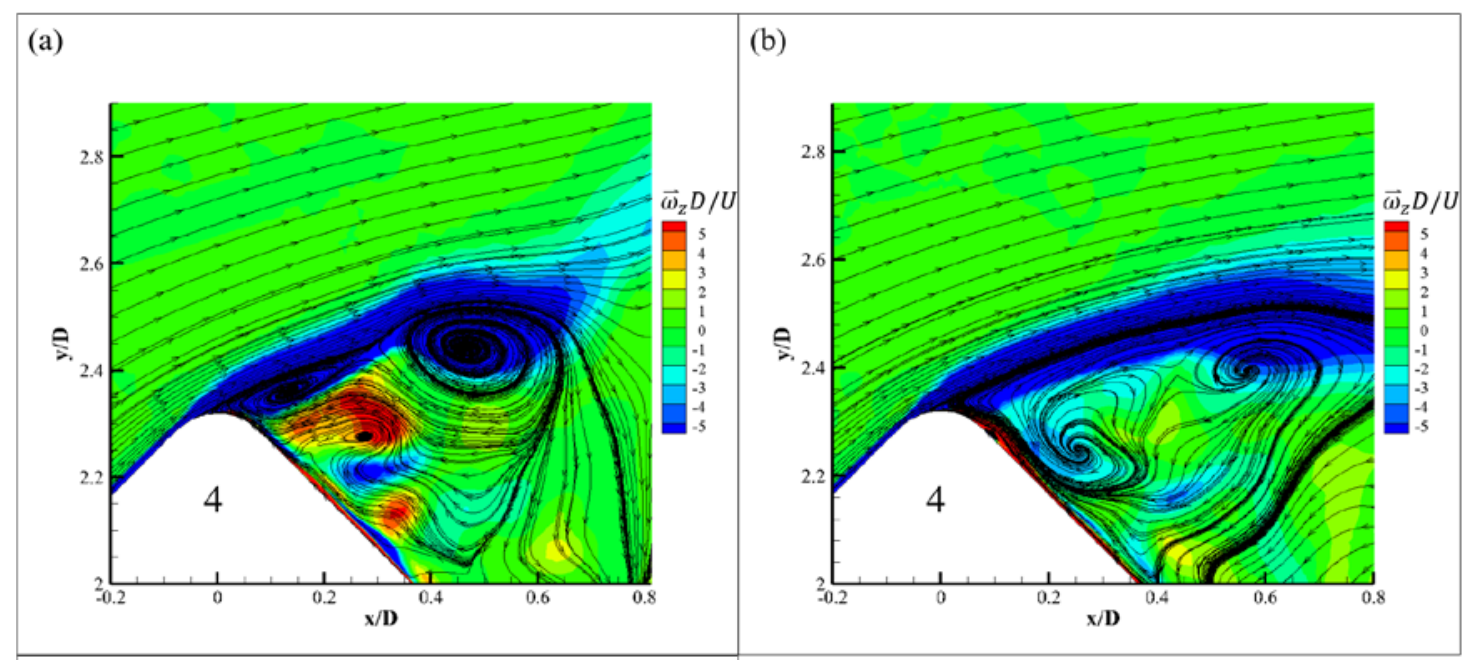

(c)

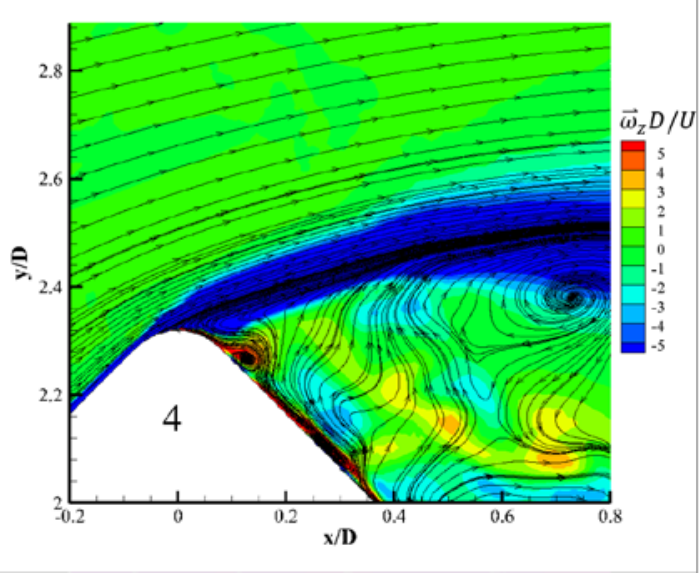

Fig. 21 The streamlines along with the vorticity $\left(\vec{\omega}_{z} D / U\right)$ contours around column 4 (the four columns with pontoons connected configuration) at different draft levels (a: middle draft level; b: $z=0.13075 \mathrm{~m}$ draft level; c: pontoon upper face draft level) showing the flow separation in $X Y$ plane at $R e=4.3 \times 10^{4}$.

The wake of column 3, which is the column in the wake region of other 3 columns, shows different flow separation patterns. As illustrated in Fig. 22 and Fig. 23, by adding the pontoons, the width of wake region in the column downstream is seen expanding compared with the column only configuration. The number of circulation regions close to the column wall also increases with the pontoons connecting between the columns. 

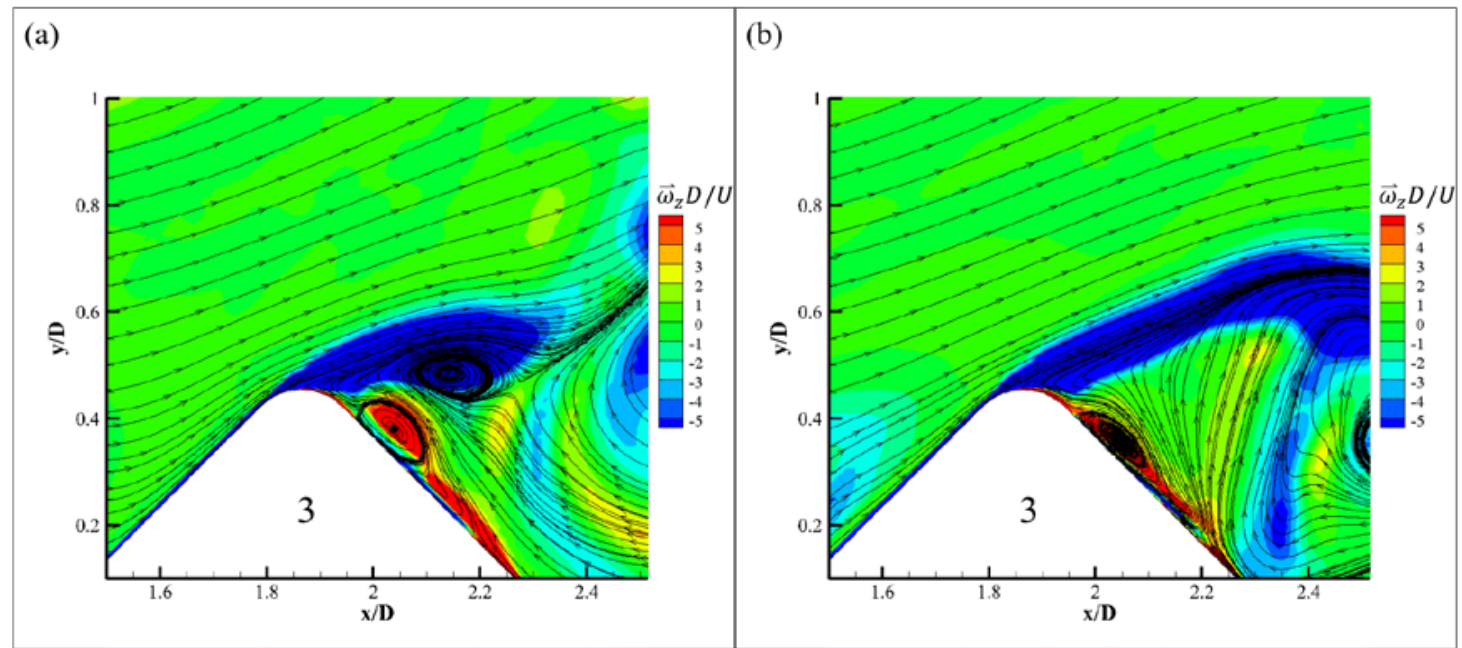

Fig. 22 The streamlines along with the vorticity $\left(\vec{\omega}_{z} D / U\right)$ contours around column 3 (the four columns
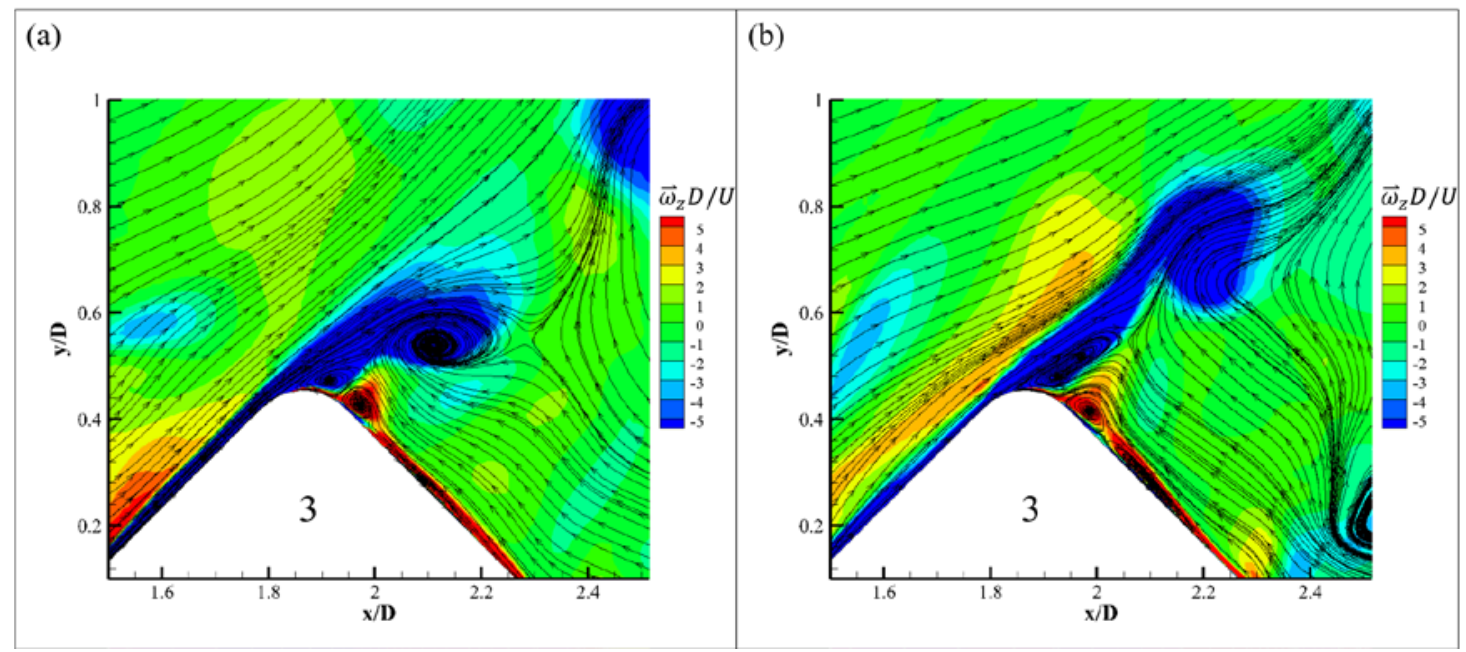

402

403

404

405

406

407

408

409

410

Fig. 23 The streamlines along with the vorticity $\left(\vec{\omega}_{z} D / U\right)$ contours around column 3 (the four columns with pontoons connected configuration) at different draft levels (a: middle draft level; b: $z=0.13075 \mathrm{~m}$ draft level) showing the flow separation in $X Y$ plane at $R e=4.3 \times 10^{4}$.

\subsubsection{Time-average pressure distribution on the surface of each column}

Fig. 24 shows the mean pressure coefficient distributions of each column at middle draft level. The pressure coefficient on the column surface is defined as:

$$
C_{p}=\frac{\left(p-p_{\infty}\right)}{\frac{1}{2} p_{\infty} U^{2}}
$$

where $p_{\infty}$ is the oncoming flow ambient pressure. 

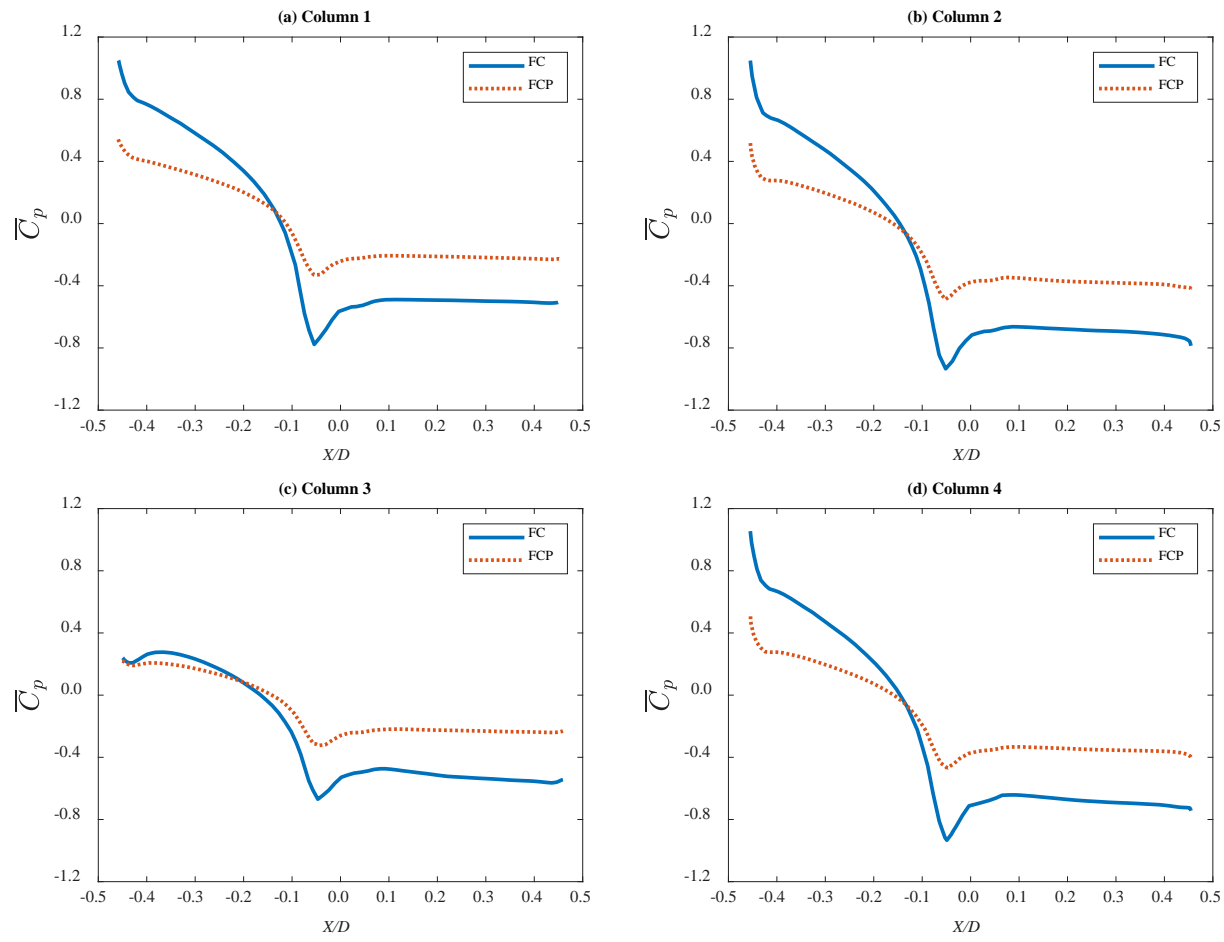

Fig. 24. Mean pressure coefficient distributions of each column at middle draft level $(X / D=0$ is the geometry centre of each column, the incoming flow pasts each column from negative to positive).

As can be seen in Fig. 24, by adding the pontoons to the structure, the pressure distribution on the surface of each column were appreciably altered. There is a clear indication that adding the pontoons to the structure resulting the pressure distribution becoming more stable compared to the four columns only configuration. In addition, the pressure distribution on Column 1, 2 and 4 show the same trend due to these three columns are directly faced to the incoming flow. Column 3, which is the downstream column, shows a different trend, the pressure distribution on the upstream face of the column reduced significantly compared with the rest 3 columns due to the wake effect.

\subsection{Flow characteristics in the vertical plane}

It is noted that the vortices shed from the free end of each column can significantly alter the vortex shedding flow region, and consequently the main contribution to the changes in the hydrodynamics between the two configurations. Thus, further study on the flow characteristics in vertical plane is warranted.

\subsubsection{Time-averaged velocity distribution}

Vertical velocity components (velocity component $k$ ) are sampled over several vertical cross sections coinciding with $X Z$ plane (i, $k$ plane) in order to investigate the differences of the downstream velocity 
428 distribution at certain offset distances $(x / D=0.25, x / D=0.75$ and $x / D=1.5)$ after each column. Herein, the 429 pontoons’ effects can be observed in Fig. 25, Fig. 26 and Fig. 27.

430 As can be seen in Fig. 25, by adding the pontoons to the structure, the vertical velocity distributions 431 have been altered significantly for the upstream column 1. There is a reduction of the velocity component $432 k$ between the two configurations. With pontoons connected, the vertical velocity decreases rapidly over the bottom level at the downstream positions of $x / D=0.25$ and $x / D=0.75$ behind column 1 . Additionally,

434 the four columns with pontoons connected configuration is observed to have a stronger recirculation compared with the four columns without pontoons configuration. The four columns configuration is shown to have a smooth velocity variation at points along the vertical direction at the position slightly behind column $1(x / D=0.75$ and $x / D=1.50)$. The same as for column 1 , clear recirculation is also evident behind the side column, column 2, for the four columns with pontoons connected configuration (see Fig. 26 at $x / D$ $=0.25)$. By adding the pontoons, the vertical velocities decrease as well. However, the differences of vertical velocity distributions are not as significant as in the case of column 1 . As can be seen in Fig. 27, for the downstream column 3, the differences between two configurations are less significant than those for

442 the three upstream columns. The four columns with pontoons connected configuration has a very smooth vertical velocity distribution behind column 3 at the position of $x / D=0.75$ and $x / D=1.50$. It is noted that

444 the four columns configuration still has the bulge between the bottom level and the pontoon upper face level. In addition, the vertical velocities of the four columns with pontoons connected configuration are higher than the vertical velocities of the four columns configuration for most part of the vertical region. 

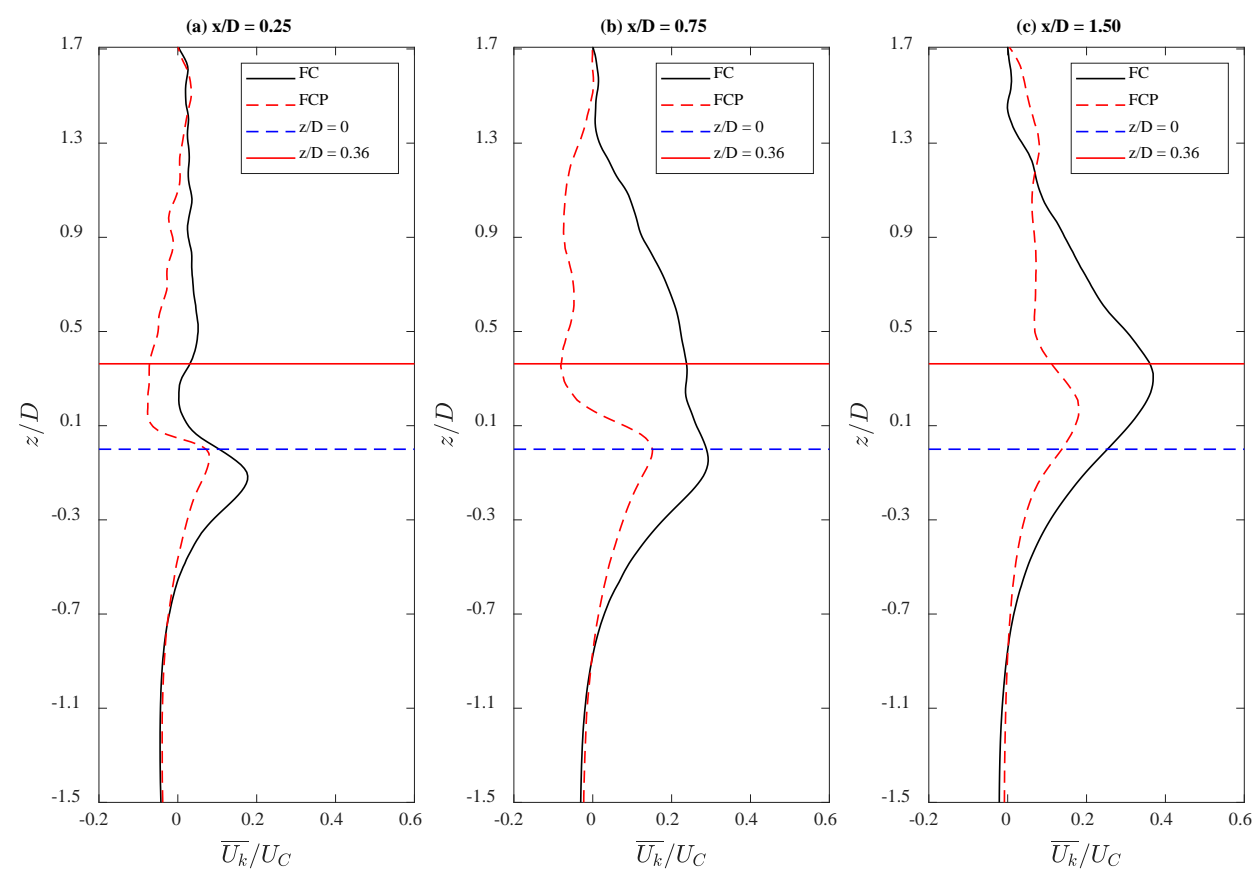

448 Fig. 25 Time-averaged flow properties of the $\overline{U_{k}} / U_{C}$ (velocity component $k$ ) in $X Z$ plane for column 1 449 with downstream distances $(x / D=0.25, x / D=0.75$ and $x / D=1.5)$ at $y / D=0$ (the central line of column), $450 R e=4.3 \times 10^{4}$. "FC" is the four columns configuration and "FCP" is the four columns with pontoons 451 connected configuration. " $z / D=0$ " is the bottom of the column and " $z / D=0.36$ " is the pontoon upper face 452 level. 

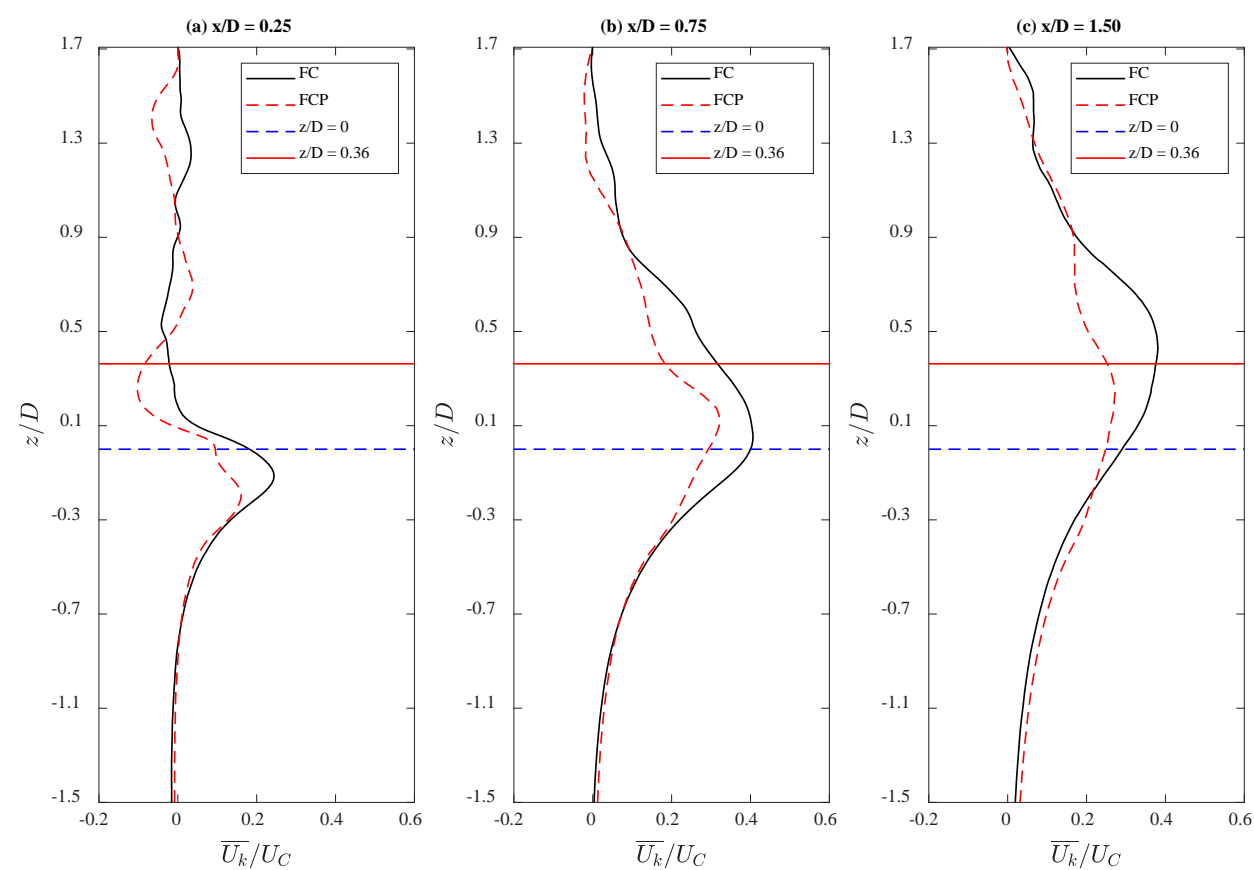

453

$454 \quad$ Fig. 26 Time-averaged flow properties of the $\overline{U_{k}} / U_{C}$ (velocity component $k$ ) in $X Z$ plane for column 2 455 with downstream distances $(x / D=0.25, x / D=0.75$ and $x / D=1.5)$ at $y / D=0$ (the central line of column), $456 R e=4.3 \times 10^{4}$. "FC" is the four columns configuration and "FCP" is the four columns with pontoons 457 connected configuration. " $z / D=0$ " is the bottom of the column and " $z / D=0.36$ " is the pontoon upper face 458 level. 

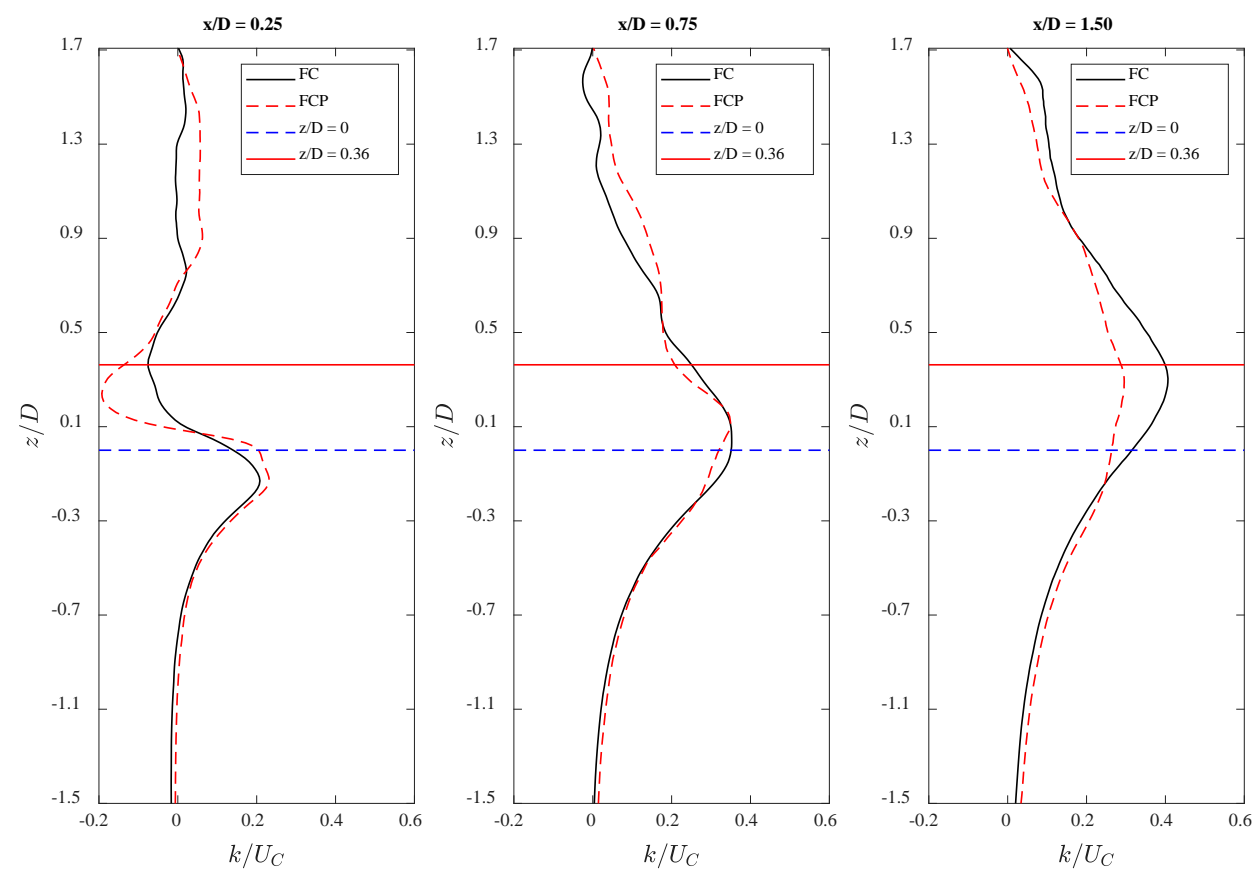

Fig. 27 Time-averaged flow properties of the $\overline{U_{k}} / U_{C}$ (velocity component $k$ ) in $X Z$ plane for column 3 with downstream distances $(x / D=0.25, x / D=0.75$ and $x / D=1.5)$ at $y / D=0$ (the central line of column), $R e=4.3 \times 10^{4}$. "FC" is the four columns configuration and "FCP" is the four columns with pontoons connected configuration. " $z / D=0$ " is the bottom of the column and " $z / D=0.36$ " is the pontoon upper face

464 level.

In addition, the streamwise velocity profiles (velocity component $i$ ) in the wake region at the vertical cross section coinciding with $Y Z$ plane at $x / D=0.75$ are sampled in order to investigate the differences of the downstream velocity distribution at a certain offset distance (where $x / D=0.75$ ) downstream each column. Herein, the pontoons' effects as well as the formation of tip and arch vortex can be observed in Fig. 28, Fig. 29 and Fig. 30.

As can be seen in Fig. 29, by adding the pontoons to the structure, the streamwise velocity distributions have been altered for the upstream column 1. The tip and arch vortex shed from the free end of column 1 have been significantly reduced, similar to column 2 \& 4 in Fig. 29 and column 3 in Fig. 30. It is also noted that by adding the pontoons to the structure, two high streamwise velocity regions have been observed at the outside corner of the pontoons (see Fig. 28 and Fig. 29). This is due to the vortices shed from the edges of pontoons which will be discussed in the following section. 
476

477

478

479

480

481

482

483

484
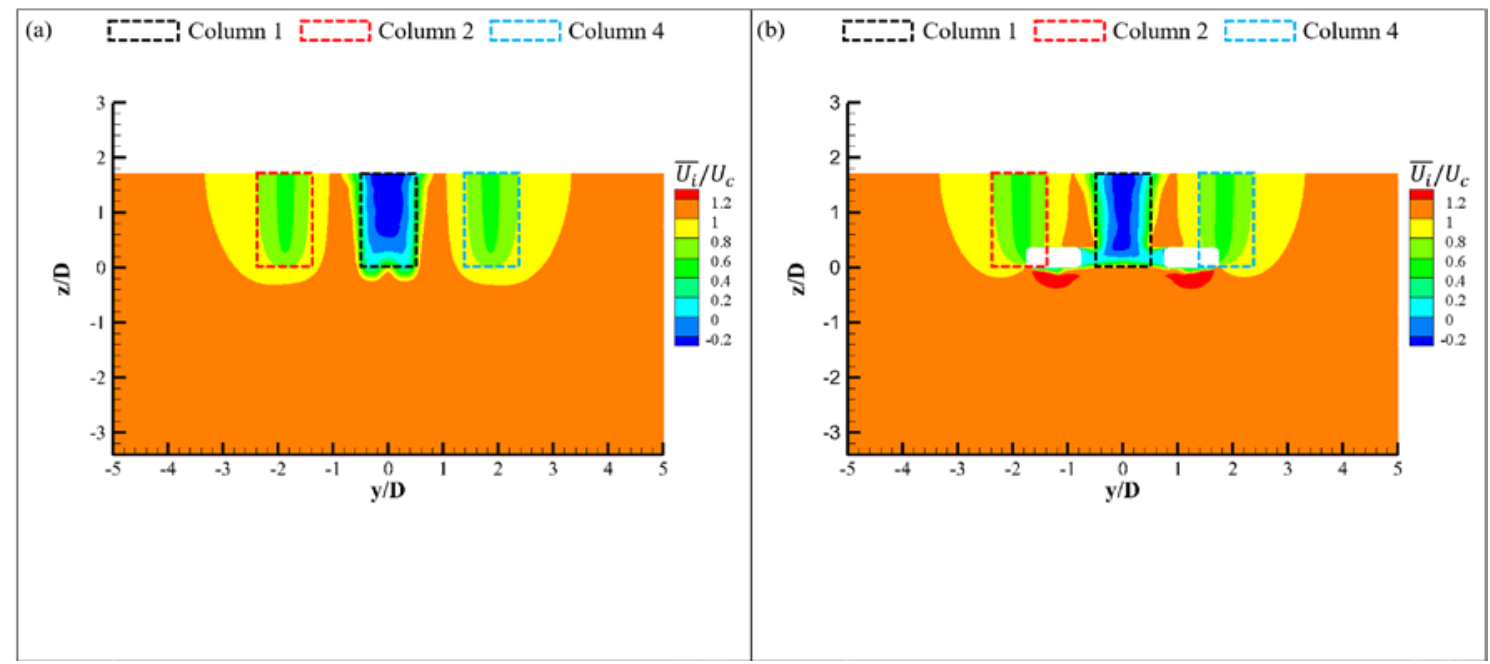

Fig. 28 Time-averaged flow properties of the $\overline{U_{i}} / U_{C}$ (velocity component $i$ ) in $Y Z$ plane for column 1 with downstream distance at $x / D=0.75, R e=4.3 \times 10^{4}$. (a) is the four columns configuration and (b) is the four columns with pontoons.

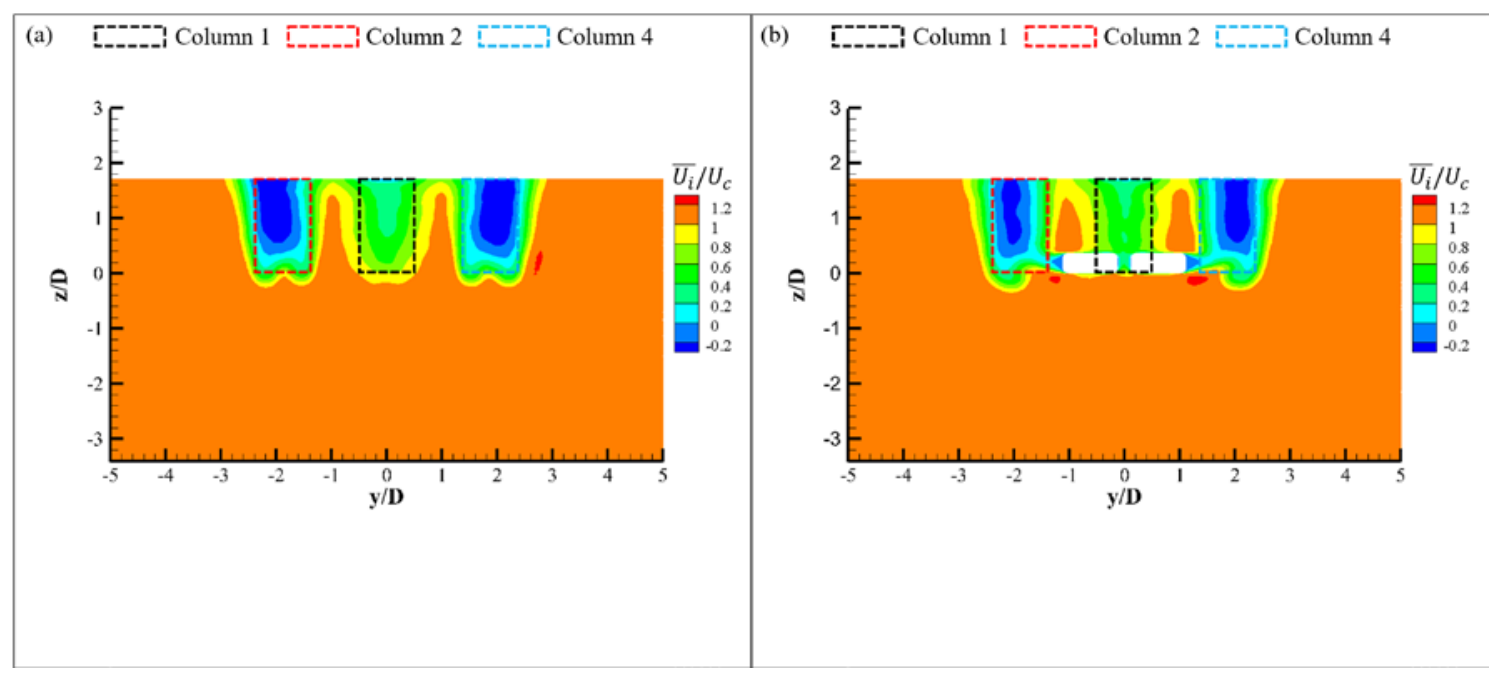

Fig. 29 Time-averaged flow properties of the $\overline{U_{i}} / U_{C}$ (velocity component $i$ ) in $Y Z$ plane for column 2 and 4 with downstream distance at $x / D=0.75, R e=4.3 \times 10^{4}$. (a) is the four columns configuration and (b) is the four columns with pontoons. 


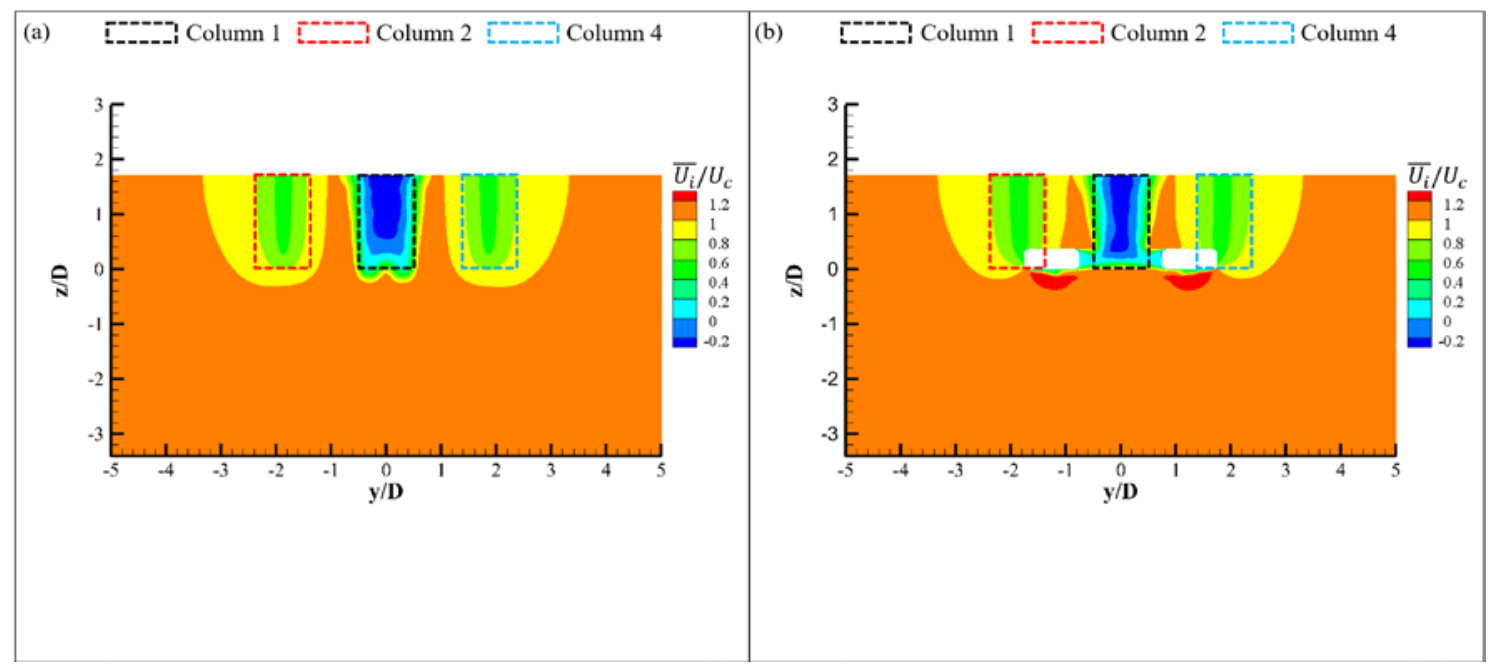

485

486

487

488

489

490

491

492

493

494

495

496

497

498

499

500

501

Fig. 30 Time-averaged flow properties of the $\overline{U_{i}} / U_{C}$ (velocity component $i$ ) in $Y Z$ plane for column 3 with downstream distance at $x / D=0.75, R e=4.3 \times 10^{4}$. (a) is the four columns configuration and (b) is the four columns with pontoons.

\subsubsection{Instantaneous vorticity contour}

In this section, the flow patterns observed in the vertical cross sections are discussed in detail to examine the free end effects on the hydrodynamics of the configurations.

For the four columns configuration, the vertical wake region behind column 1 is quite similar to that developing in the horizontal wake region. The wake is fully occupied with fragmentized vortices, as can be clearly seen in Fig. 31. Unlike column 1, column 3 has a clearer organised and tidy wake region, where only one obvious strong vortex can be seen (Fig. 31). In addition, a small portion of the vortices shed from the free end of column 1 is seen joining into the column 3 free end shear layer (red circled in Fig. 31(a) and Fig. 31(b)). Parts of the vortices that have been shed from the free end of column 1 appear to recirculate and impinge back on column 1. Majority of the vortices shed from column 1 impinge on the incidence flow of column 3, which are red circled in Fig. 31(c) and Fig. 31(d). For the side column (column 2, see Fig. 32), a quite similar flow pattern is shown to that induced by a single finite column does [16], and this is expected since there is no blocked structure behind this column. 


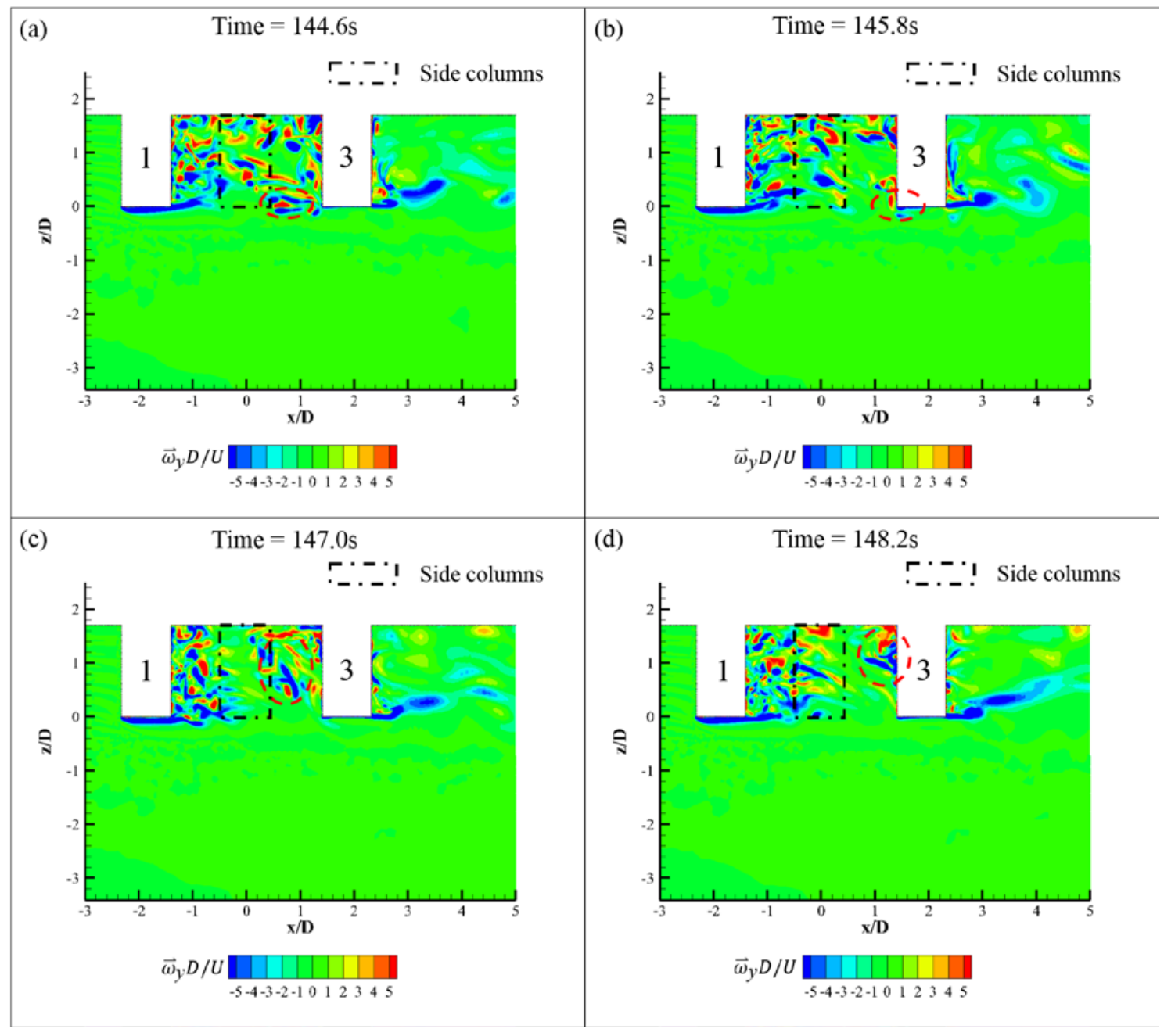

Fig. 31 A time series of the non-dimensional transverse vorticity $\left(\vec{\omega}_{y} D / U\right)$ contours around the four

504 columns configuration at the central line of column 1 and 3 showing the instantaneous flow fields in $X Z$ 505 plane at $R e=4.3 \times 10^{4}$. 


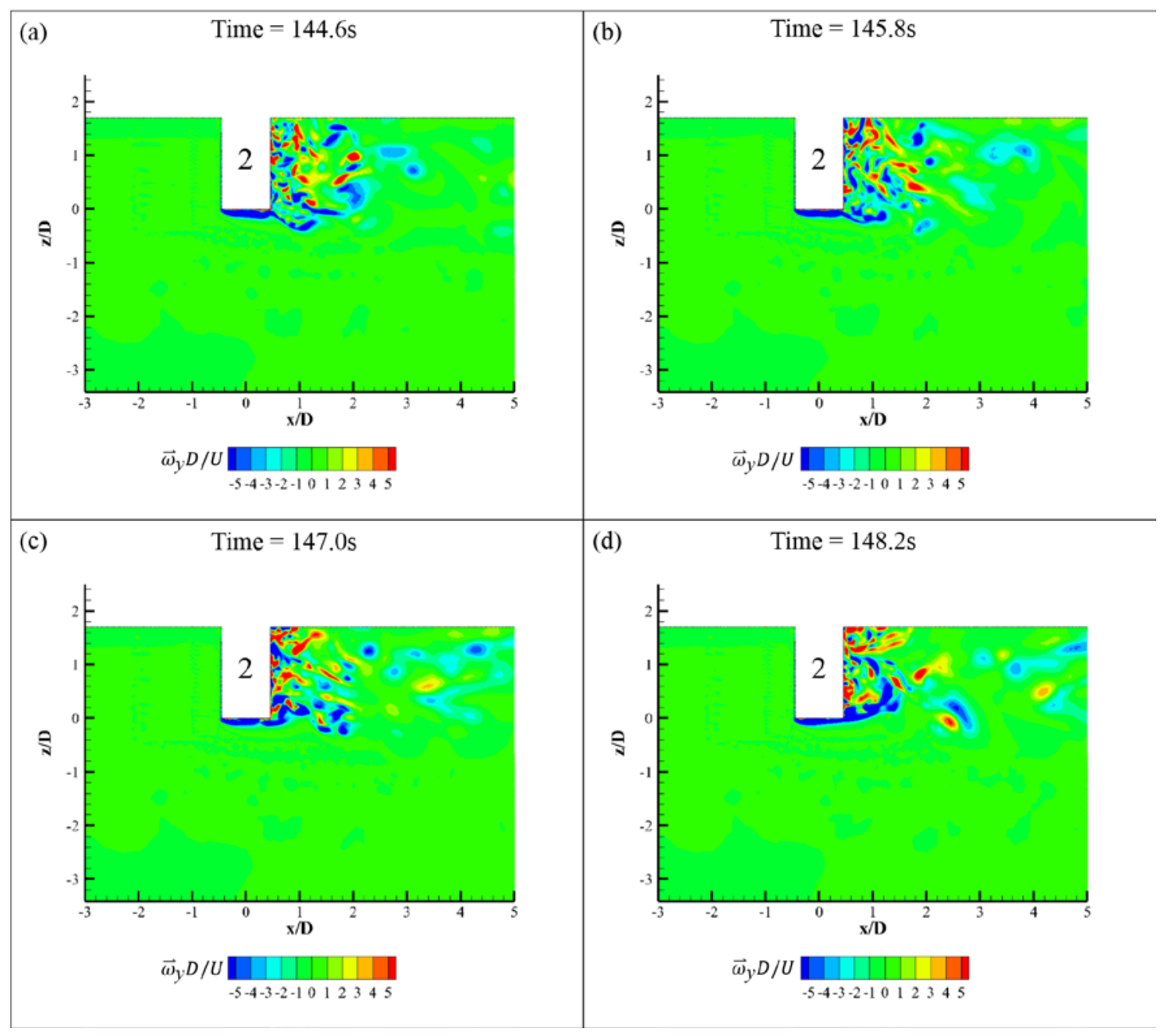

Fig. 32 A time series of the non-dimensional transverse vorticity $\left(\vec{\omega}_{y} D / U\right)$ contours around the four columns configuration at the central line of column 2 showing the instantaneous flow fields in $X Z$ plane at $\operatorname{Re}=4.3 \times 10^{4}$.

510 With the pontoons being added to the structure, significant change can be observed in the vertical plane

511 flow patterns around each column (Fig. 33). For column 1, the vortices shed from the free end become

512 slimmer compared with the corresponding vortices from the four columns configuration. Additionally, the

513 amount of the fragmentized vortices behind column 1 was reduced by adding the pontoons. Unlike the four

514 columns configuration, the majority of the vortices shed from the free end of column 1 are seen joining

515 with the vortices being shed from the free end of column 3 (see red circled part in Fig. 33(c) and Fig. 33(d)).

516 This vortex mixing behaviour makes the vortices behind column 3 to be shorter than those in the four

517 columns configuration. For the side columns (e.g. column 2), by connecting the pontoons between the columns, the vortex shedding behaviour at the free end completely changed. As shown in Fig. 34, two 
519 vortices (red and black circled in Fig. 34(a)) are shed from the separate edges of the free end with one

520 quickly mixing with the vortices being shed from the side of the column and the other tends to remain at a

521 certain vertical level for a period of time and then amalgamate into the wake region with the vortices shed 522 from the sides of the same column.

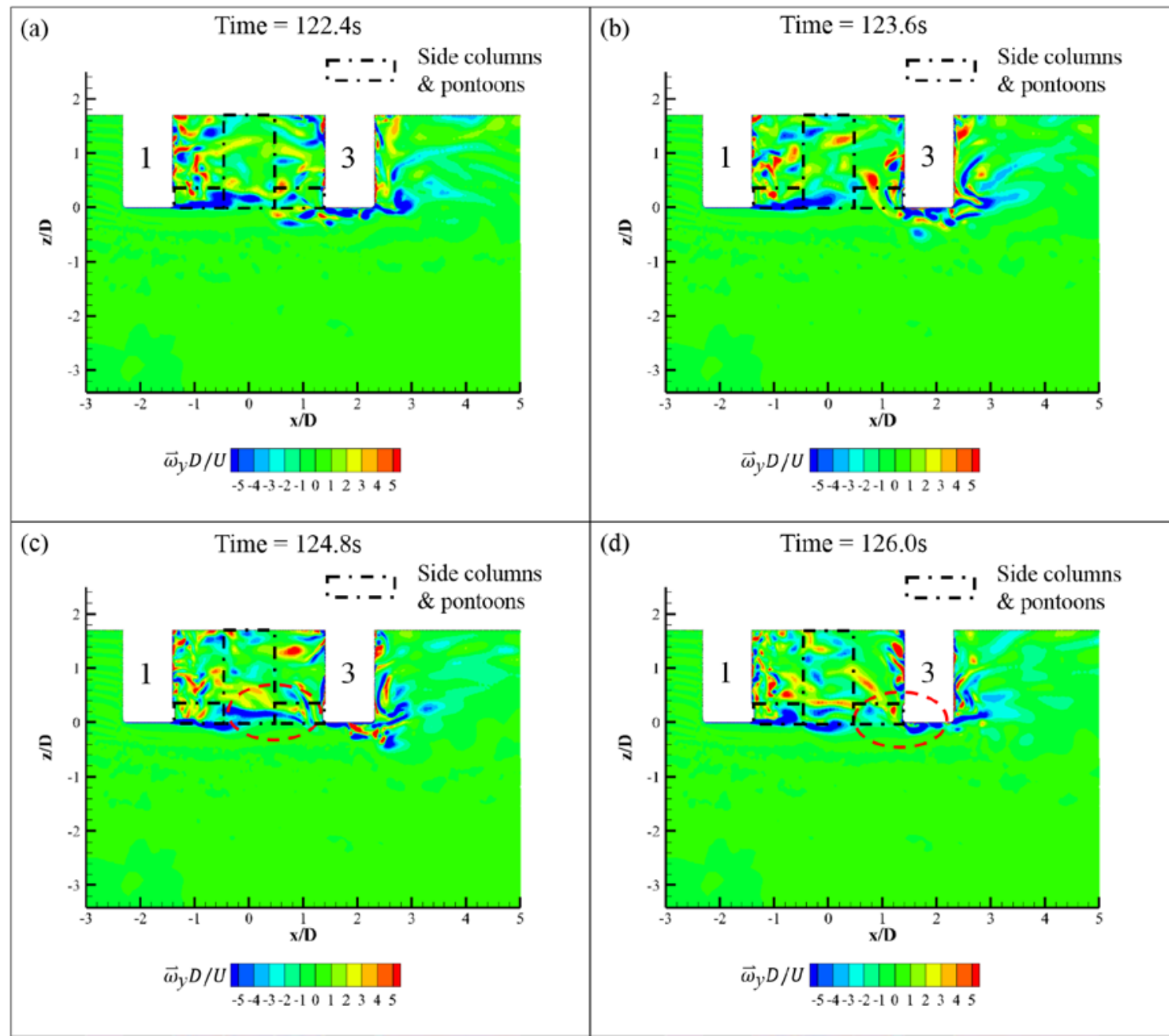

524 Fig. 33 A time series of the non-dimensional transverse vorticity $\left(\vec{\omega}_{y} D / U\right)$ contours around the four 525 columns with pontoons connected configuration at the central line of column 1 and 3 showing the instantaneous flow fields in $X Z$ plane at $R e=4.3 \times 10^{4}$. 


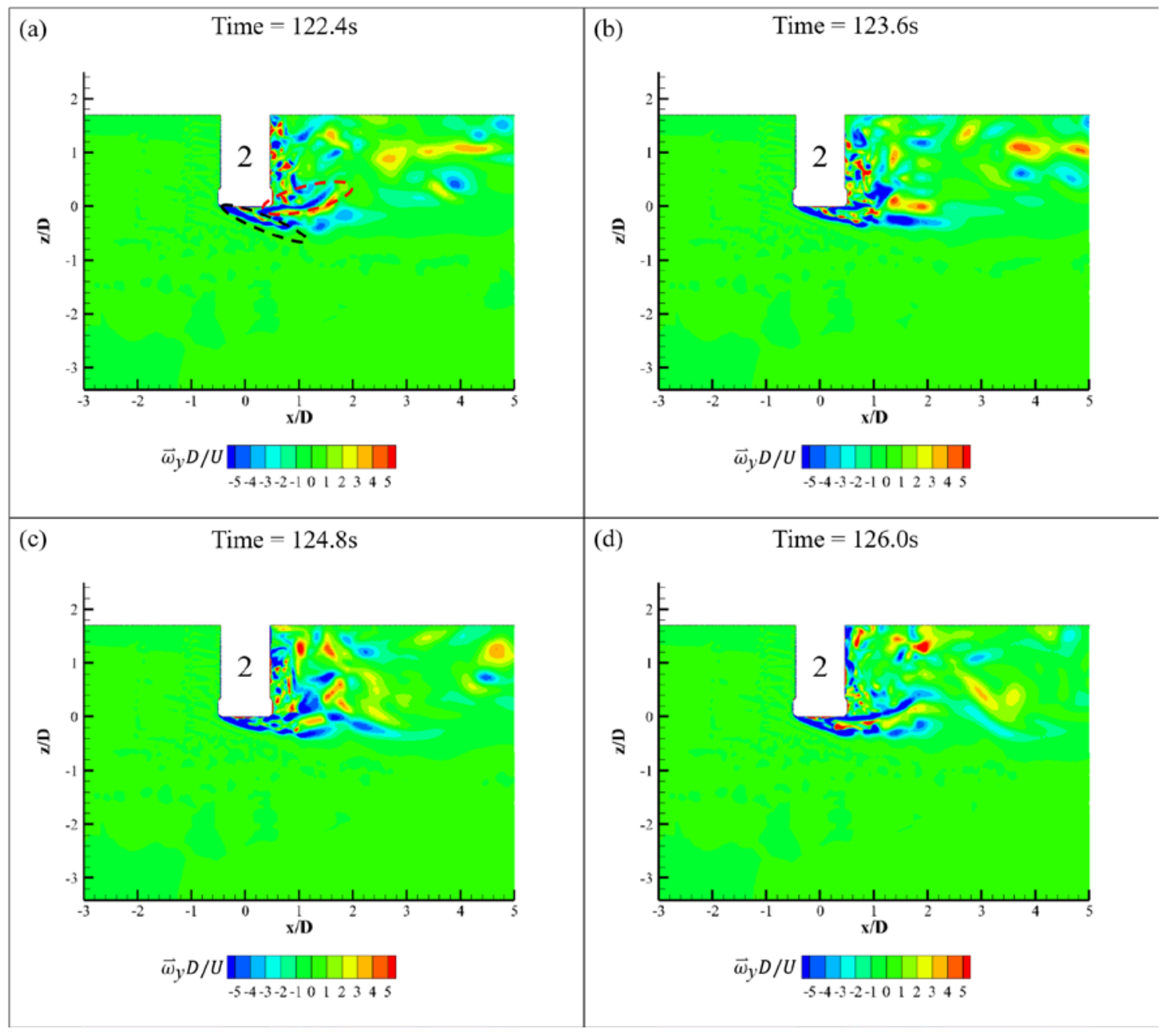

Fig. 34 A time series of the non-dimensional transverse vorticity $\left(\vec{\omega}_{y} D / U\right)$ contours around the four columns with pontoons connected configuration at the central line of column 2 showing the instantaneous flow fields in $X Z$ plane at $R e=4.3 \times 10^{4}$.

\subsection{Flow characteristics of instantaneous vorticity isosurfaces}

The instantaneous vorticity isosurfaces have also been closely related to the fluid physics, especially in the vortex flow. It is a surface that represents points of a constant value of non-dimensional spanwise vorticity in three-dimensional space. Thus, the 3D characteristics of vortex shedding process is further analysed and demonstrated in this section.

In order to examine the overall flow patterns in further detail, the vorticity isosurface obtained from the numerical simulations for the two different configurations are plotted in Fig. 35 and Fig. 36. As shown in

Fig. 35, a constant value of non-dimensional spanwise vorticity $\left(\vec{\omega}_{z} D / U=1.5\right)$ in 3D space has been shown in $X Y$ plane. For the four columns configuration, there are a large number of small vortices in the wake 
540 downstream. However, by adding the pontoons to the structure, the flow characteristics were appreciably

541 altered. As shown in Fig. 36, the vortices becoming more stable compared to the four columns only 542 configuration. Similar to the observation obtained in Fig. 18 and Fig. 19. It also can be seen in Fig. 36,

543 there are a number of small vortices shed from the pontoons which affect the downstream wake region. To

544 quantify the influence, the width of the wake region, a linear outer contour line of the isosurfaces has been

545 calculated and plotted in the figures (shown as the red lines in Fig. 35 and Fig. 36). The outer contour line

546 was firstly extracted from the outside point at each vortices shed from the portside column and further fitted

547 as a linear regression line. In the present study, the slope ratio was obtained by averaging the linear outer

548 contour line within one vortex shedding period. This ratio can generally describe the width of the wake

549 region. For the four columns configuration, the absolute value of slope ratio is 0.203 . By adding the

550 pontoons to the structure, the absolute value of slope ratio increases to 0.273 , presenting an approximately

$55134.5 \%$ increase in this instance. Therefore, it is a clear evidence that the wake region is expanded as a

552 consequence of adding the pontoons to the structure. 

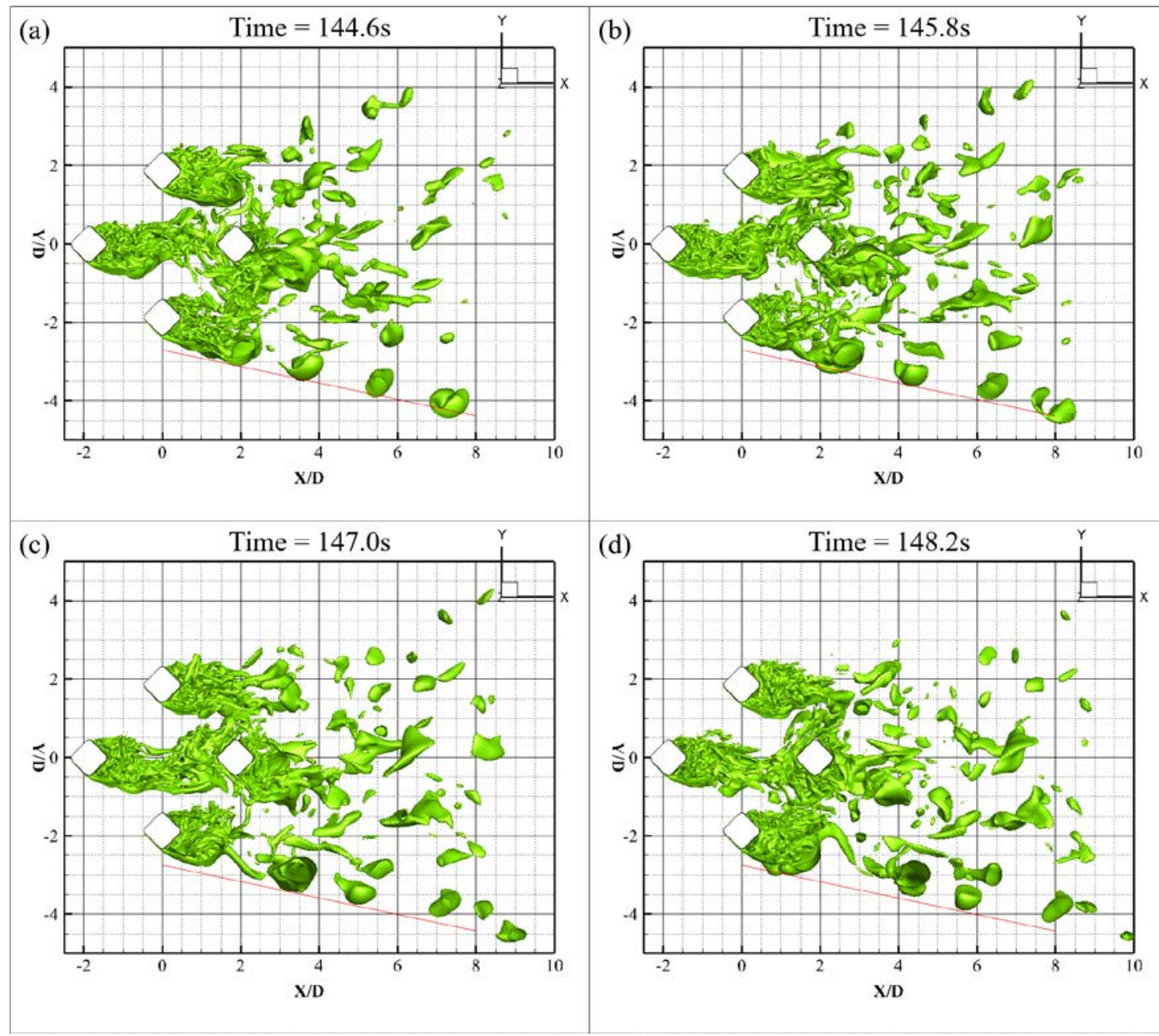

$554 \quad$ Fig. 35 A time series of the non-dimensional spanwise vorticity $\left(\vec{\omega}_{z} D / U=1.5\right)$ isosurfaces around the

555 four columns configuration showing the instantaneous flow fields at $R e=4.3 \times 10^{4}$. The red line is the average outside line within one vortex shedding period showing the linear outer contour line of the 557 isosurfaces. 

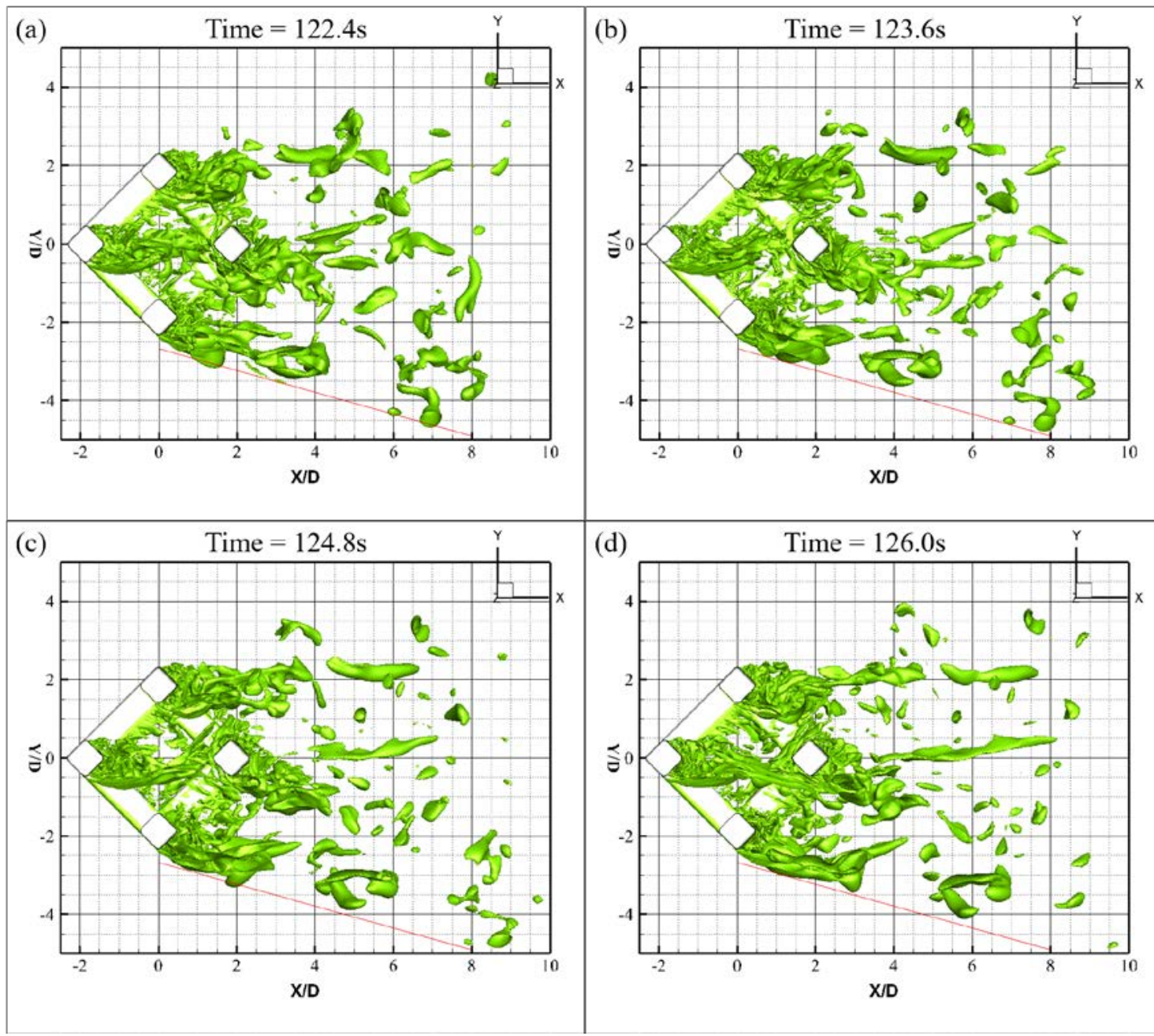

Fig. 36 A time series of the non-dimensional spanwise vorticity $\left(\vec{\omega}_{z} D / U=1.5\right)$ isosurfaces around the four columns with pontoons connected configuration showing the instantaneous flow fields at $R e=4.3 \times 10^{4}$. The red line is the average outside line within one vortex shedding period showing the linear outer contour line of the isosurfaces.

To understand the vertical structures of the wake regions associated with the two configurations, a vortex identification method based on the Q-criterion [30] has been employed in the present study. Fig. 37 and Fig. 38 present the Q-criterion based vertical structures for the two configurations with and without pontoons connected. The isofurfaces are shown at a constant positive value where $Q=1$. By connecting the pontoons between the columns, the vortex shedding behaviour at the free end significantly changed with the most striking change being that the vortices can be rarely observed at the end of the upstream column (column 1) in Fig. 38. In addition, by adding the pontoons to the structures, the overall wake region of the whole structures become tidier and more clearly defined. Slender vortices can be seen in Fig. $\mathbf{3 8 .}$ 
571 However, the whole flow region around the four columns configuration are fully occupied with 572 fragmentized vortices as shown in Fig. 37.

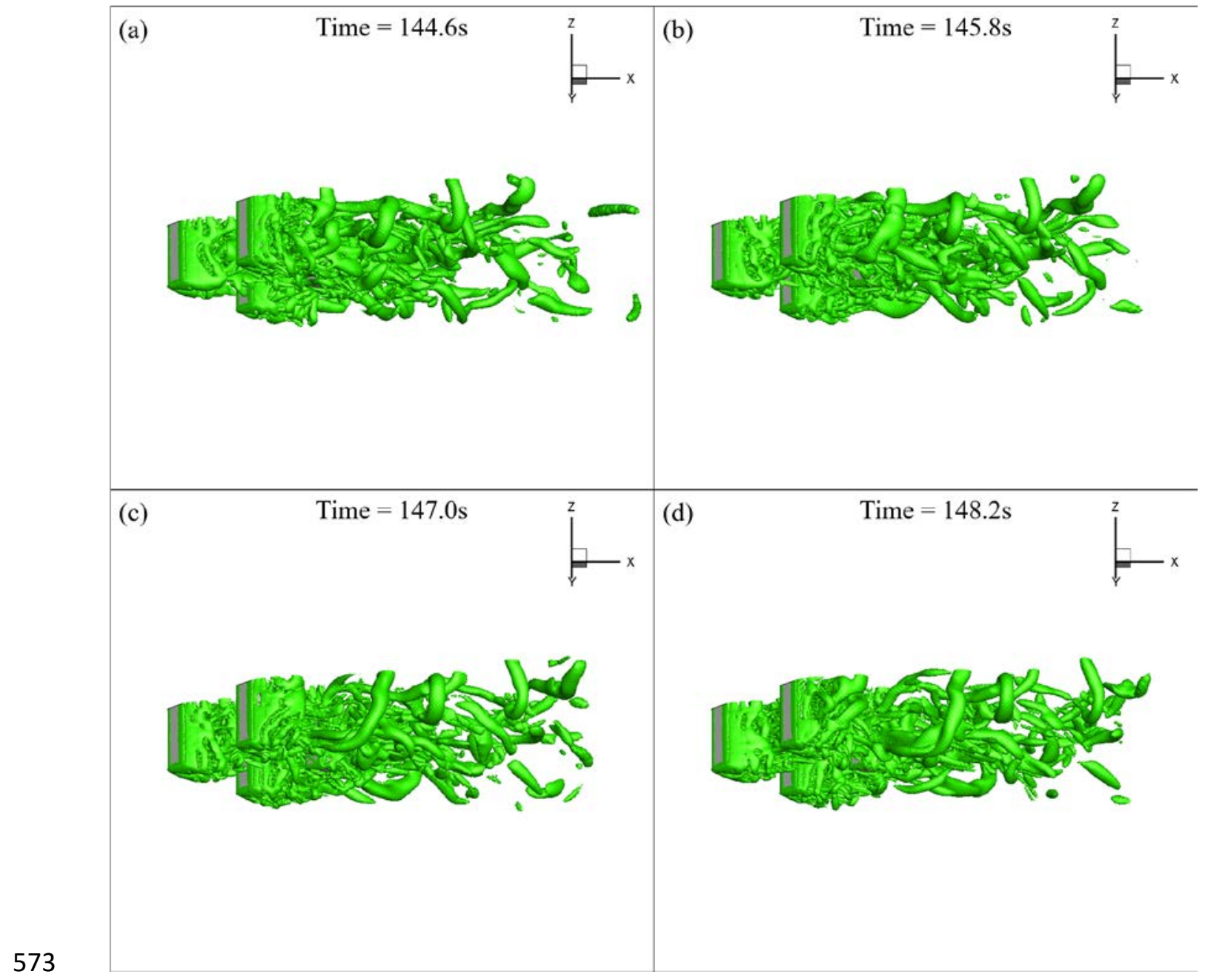

574 Fig. 37 A time series of isometric view representation of $Q$-criterion of the four columns configuration 575 with $Q=1$, showing the instantaneous flow fields at $R e=4.3 \times 10^{4}$. 


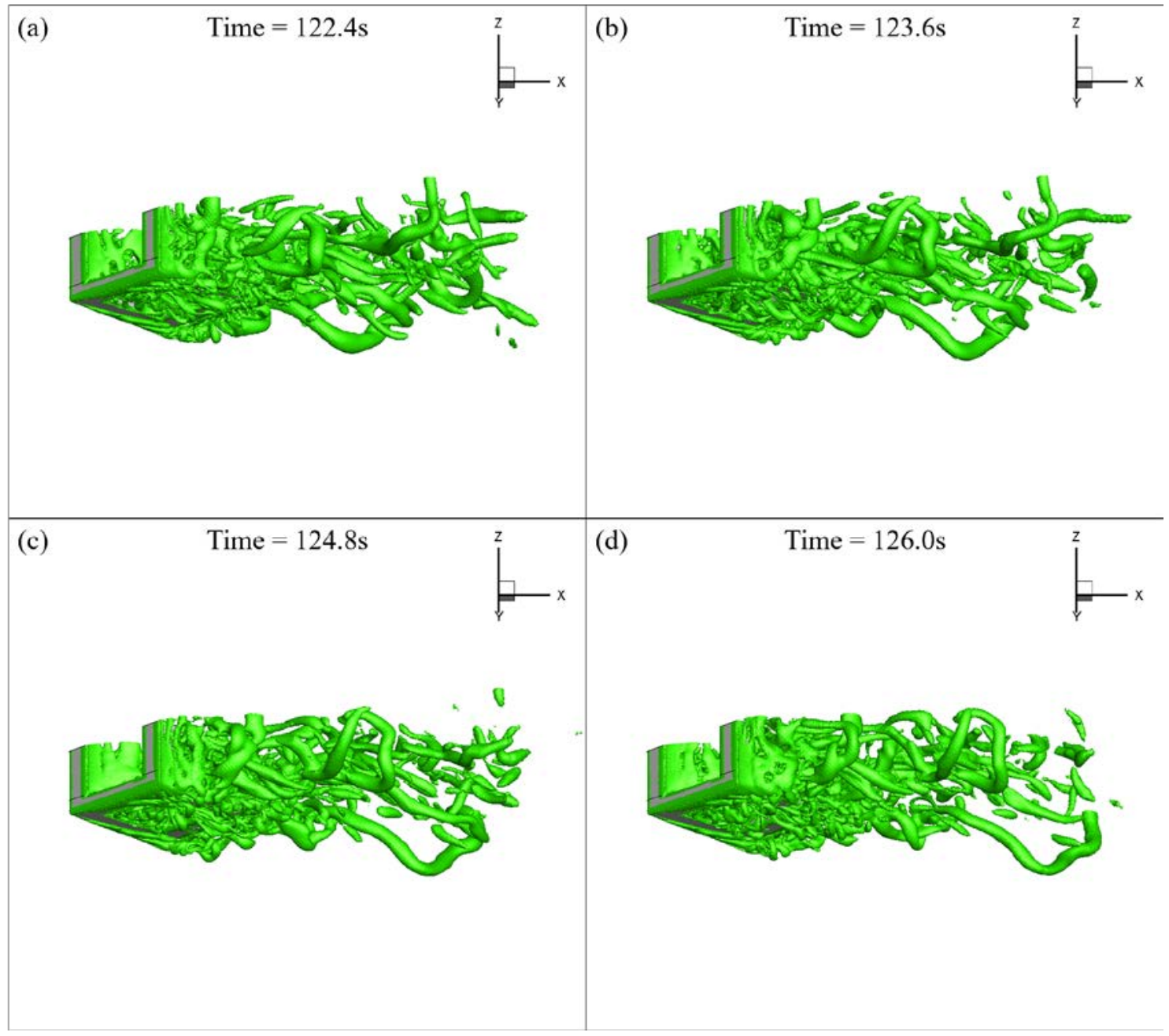

$577 \quad$ Fig. 38 A time series of isometric view representation of $Q$-criterion of the four columns with pontoons

578 connected configuration with $Q=1$, showing the instantaneous flow fields at $R e=4.3 \times 10^{4}$.

579 It should be pointed out that both configurations are fixed in the present experimental and numerical 580 study. It is reported that considerable alteration in vortex flow characteristics occurred if the structural 581 configurations undergo the so called vortex-induced-motion (VIM) [26, 31]. In their experimental and 582 numerical study, Liu et al. [31] demonstrated that the VIM of a deep-draft semi-submersible can be significantly reduced due to the vortex shedding process being suppressed by adding the pontoons. Liang

584 and Tao [26] demonstrated the negative work done by pontoons while the work done by the columns being dominantly positive during VIM.

\section{4.4. Overall drag and lift forces}



numerical simulations are shown in this section.

Table 6 The resulting non-dimensional coefficients $\bar{C}_{D}, C_{L r m s}$ and $S t$ for the four columns configuration (“num.” represents "numerical”; “exp.” represents "experimental”).

\begin{tabular}{lllllll}
\hline \hline$R e$ & $\bar{C}_{D}$ (num.) & $\bar{C}_{D}$ (exp.) & $C_{\text {Lrms }}$ (num.) & $C_{\text {Lrms }}$ (exp.) & $S t$ (num.) & $S t$ (exp.) \\
\hline $3.7 \times 10^{4}$ & 1.083 & $0.948 \pm 0.002$ & 0.062 & $0.053 \pm 0.002$ & 0.156 & $0.137 \pm 0.004$ \\
$4.3 \times 10^{4}$ & 1.075 & $0.961 \pm 0.009$ & 0.066 & $0.055 \pm 0.001$ & 0.152 & $0.139 \pm 0.002$ \\
$5.2 \times 10^{4}$ & 1.077 & $0.962 \pm 0.009$ & 0.066 & $0.051 \pm 0.001$ & 0.150 & $0.139 \pm 0.001$ \\
$6.0 \times 10^{4}$ & 1.068 & $0.990 \pm 0.005$ & 0.066 & $0.053 \pm 0.002$ & 0.151 & $0.141 \pm 0.002$ \\
\hline \hline
\end{tabular}

Table 7 The resulting non-dimensional coefficients $\bar{C}_{D}, C_{\text {Lrms }}$ and $S t$ for the four columns with pontoons connected configuration (“num.” represents "numerical”; “exp.” represents "experimental”).

\begin{tabular}{lllllll}
\hline \hline$R e$ & $\bar{C}_{D}$ (num.) & $\bar{C}_{D}$ (exp.) & $C_{\text {Lrms }}$ (num.) & $C_{\text {Lrms }}$ (exp.) & St (num.) & St (exp.) \\
\hline $3.7 \times 10^{4}$ & 1.054 & $0.932 \pm 0.008$ & 0.070 & $0.078 \pm 0.002$ & 0.132 & $0.123 \pm 0.001$ \\
$4.3 \times 10^{4}$ & 1.043 & $0.940 \pm 0.015$ & 0.072 & $0.078 \pm 0.009$ & 0.139 & $0.122 \pm 0.004$ \\
$5.2 \times 10^{4}$ & 1.047 & $0.953 \pm 0.008$ & 0.071 & $0.076 \pm 0.005$ & 0.142 & $0.124 \pm 0.005$ \\
$6.0 \times 10^{4}$ & 1.051 & $0.974 \pm 0.005$ & 0.078 & $0.082 \pm 0.004$ & 0.139 & $0.126 \pm 0.000$ \\
\hline \hline
\end{tabular}

As can been in Table 6 and Table 7, the overall hydrodynamics remain relatively stable in the Reynolds number range examined. In addition, with the aim to illustrate any level of uncertainty in the experimental measurements, the error bounds are illustrated in each table.

\subsection{Drag and lift force on each structural member}

In order to further examine vortex flow dynamics on the structure and to improve the understanding of interactions between the vortex shedding processes due to each of the columns of the structures, the hydrodynamic force coefficients on each individual column of the two configurations are further calculated in the numerical simulations and the results are presented in Fig. 39, Fig. 40 and Fig. 41.

The mean drag force coefficient (see Fig. 39) on each column remains stable within the current 603 Reynolds number range and is similar to the trend of the overall $\bar{C}_{D}$. The two side columns (column 2 and 4) experience the largest $\bar{C}_{D}$ among the four columns. The upstream column (column 1) experiences a slightly smaller $\bar{C}_{D}$ than the $\bar{C}_{D}$ of the two side columns but is still significantly higher than the value of 
607 decreases slightly when the pontoons were added into the structure, however the drag force coefficient on

608 the portside and starboard side columns (column 2 and 4) increases. For the $C_{\text {Lrms }}$, the upstream column

609 has the smallest value among the four columns for both configurations. As the portside and starboard side

610 columns (column 2 and 4) are symmetrically exposed to the flow, the fluctuating lift forces on them are

611 also symmetric (as shown in Fig. 40 and Fig. 41). However, there is a significant increment for the $C_{\text {Lrms }}$

612 on the two side columns between the two configurations owing to the pontoons. It is evident that the

613 upstream column experience a large increment by adding the pontoons as shown in Fig. 40. It is noted that

614 the downstream column experiences the largest fluctuating lift force coefficient than other columns.

615 Although the root mean square lift force coefficients on the downstream column fluctuate considerably, the

616 lift force coefficient of the overall structure remains at a stable level within current Reynolds number range

617 examined. It is also revealed that the lift force coefficient on the three upstream columns (column 1, 2, and

618 4) increase markedly by adding the pontoons into the structure while the increase of the lift forces

619 coefficient on column 3 appears to be less pronounced. It is noted that a slight increase in the $C_{L r m s}$ value

620 of the overall structure is attributed to the effects of adding the pontoons as well as the phase difference of

621 the four columns.

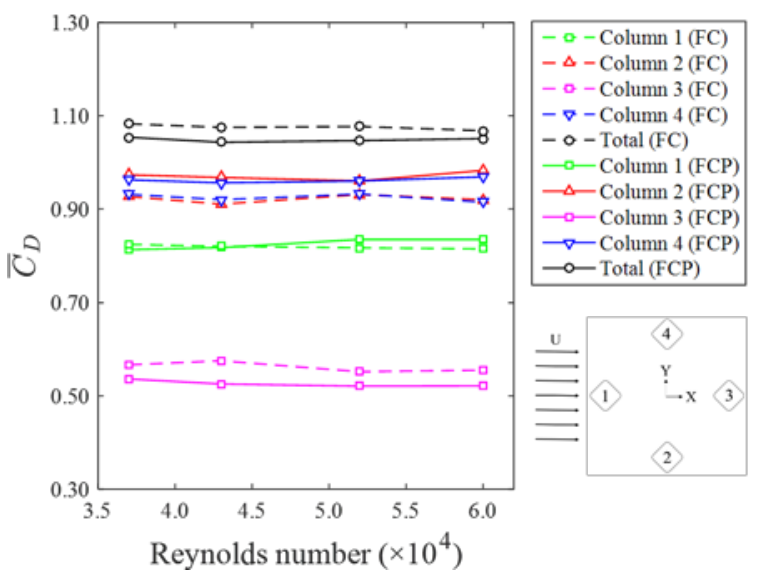

Fig. 39 Mean drag coefficients $\left(\bar{C}_{D}\right)$ on each member of the models (FC represents the four columns configuration; FCP represents the four columns with pontoons connected configuration). 


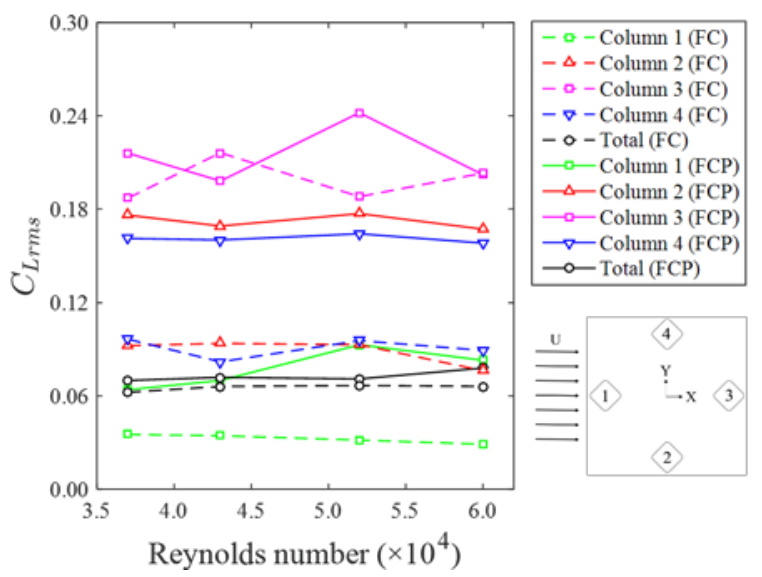

626 Fig. 40 Root mean square lift coefficients $\left(C_{L r m s}\right)$ on each member of the models (FC represents the four columns configuration; FCP represents the four columns with pontoons connected configuration).

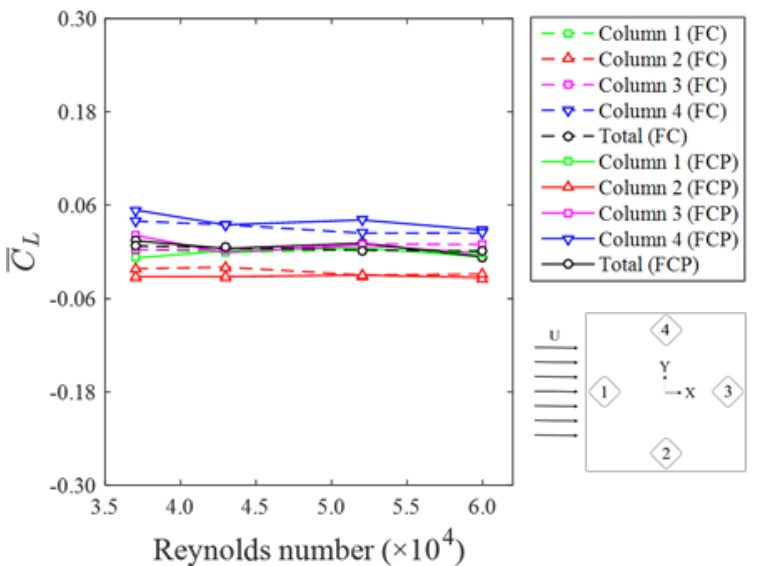

Fig. 41 Mean lift coefficient $\left(\bar{C}_{L}\right)$ on each member of the models (FC represents the four columns configuration; FCP represents the four columns with pontoons connected configuration).

\section{Conclusions}

This paper presents an experimental and numerical study focusing on the vortex shedding flow 633 interactions around multi-columns structures. While model tests conducted in a circulating channel served as a reliable benchmark for validating the numerical model, they also provided comprehensive measurements on the flow patterns by employing the particle image velocimetry (PIV) into the experiments and the hydrodynamic forces acting on the structure. Numerical simulations, on the other hand, provided substantial details on the vortex shedding characteristics as the experimental measuring range was itself quite limited. 

between the two configurations (with/without pontoons connected) are investigated in detail.

642 By examining the flow patterns at the time instantaneous from both the experimental and the numerical results, it is revealed that adding the pontoons to the structure can serve to organise and structure the vortices

644 shed from the columns well and lead the overall wake region tidy and more clearly defined. The pontoons 645 connected between each column tend to block the vortices shed from the free end of the columns, especially pushing the recirculation region further away from the free end of each column and expanding the overall

647 wake region. Difference in the wake region indicate that adding the pontoons to a basic multi-columns structure makes the vortex street more tidy and structured. Hence, the vortex shedding period and lift force are increased.

650 The analyses of the hydrodynamic force coefficients on the structures revealed that adding the pontoons to the structure had a large effect on the fluctuating force coefficients, especially for the force coefficients on the side columns. However, the influence on the drag force coefficient is not as significant as that on the lift force coefficient.

654 This study focuses on the 45 degree flow incidence, hence more incidences should be considered and examined in order to obtain a more generalized understanding on the interaction of vortex shedding processes on the flow around four columns both with and without pontoons connected.

\section{Acknowledgements}

658 The authors would like to acknowledge the support of Newton Fund of Royal Academy of Engineering 659 UK (NRCP/1415/211) and the National Natural Science Foundation of China (Grant No.51279104). This work made use of the facilities of N8 HPC Centre of Excellence, provided and funded by the N8 consortium and EPSRC (Grant No. EP/K000225/1).

\section{Reference}

[1] Thom A. The flow past circular cylinders at low speeds. Proceedings of the Royal Society of London Series A, Containing Papers of a Mathematical and Physical Character. 1933:651-69. 

and Structures. 2003;17:57-96.

[3] Parnaudeau P, Carlier J, Heitz D, Lamballais E. Experimental and numerical studies of the flow over a circular cylinder at Reynolds number 3900. Physics of Fluids. 2008;20:085101.

[4] Bearman PW. Circular cylinder wakes and vortex-induced vibrations. Journal of Fluids and Structures. 2011;27:648-58.

[5] Sayers AT. Flow interference between four equispaced cylinders when subjected to a cross flow. Journal of Wind Engineering and Industrial Aerodynamics. 1988;31:9-28.

[6] Sayers AT. Vortex shedding from groups of three and four equispaced cylinders situated in a cross flow. Journal of Wind Engineering and Industrial Aerodynamics. 1990;34:213-21.

[7] Lam K, Lo SC. A visualization study of cross-flow around four cylinders in a square configuration. Journal of Fluids and Structures. 1992;6:109-31.

[8] Lam K, Fang X. The effect of interference of four equispaced cylinders in cross flow on pressure and force coefficients. Journal of Fluids and Structures. 1995;9:195-214.

[9] Lam K, Li JY, Chan KT, So RMC. Flow pattern and velocity field distribution of cross-flow around four cylinders in a square configuration at a low Reynolds number. Journal of Fluids and Structures. 2003a;17:665-79.

682

[10] Lam K, Li JY, So RMC. Force coefficients and Strouhal numbers of four cylinders in cross flow. Journal of Fluids and Structures. 2003b;18:305-24.

[11] Lam K, Gong WQ, So RMC. Numerical simulation of cross-flow around four cylinders in an inline square configuration. Journal of Fluids and Structures. 2008;24:34-57.

[12] Lam K, Zou L. Three-dimensional numerical simulations of cross-flow around four cylinders in an in-line square configuration. Journal of Fluids and Structures. 2010;26:482-502.

[13] Zou L, Lin Y-f, Lam K. Large-eddy simulation of flow around cylinder arrays at a subcritical Reynolds number. Journal of Hydrodynamics, Ser B. 2008;20:403-13.

[14] Zhao M, Cheng L. Numerical simulation of vortex-induced vibration of four circular cylinders in a square configuration. Journal of Fluids and Structures. 2012;31:125-40. configuration. Journal of Fluids and Structures. 2013;43:179-99. 
[16] Sumner D. Flow above the free end of a surface-mounted finite-height circular cylinder: a review. Journal of Fluids and Structures. 2013;43:41-63.

[17] Morton C, Yarusevych S. Vortex shedding in the wake of a step cylinder. Physics of Fluids. 2010;22:083602.

[18] Liang Y, Tao L, Xiao L, Liu M. Experimental and Numerical Study on Flow Past Four Rectangular Columns in Diamond Configuration. ASME 2016 35th International Conference on Ocean, Offshore and Arctic Engineering: American Society of Mechanical Engineers; 2016. p. V001T01A44-VT01A44.

[19] Shur ML, Spalart PR, Strelets MK, Travin AK. A hybrid RANS-LES approach with delayed-DES and wall-modelled LES capabilities. International Journal of Heat and Fluid Flow. 2008;29:1638-49.

[20] Spalart PR, Jou WH, Strelets M, Allmaras SR. Comments on the feasibility of LES for wings, and on a hybrid RANS/LES approach. Advances in DNS/LES. 1997;1:4-8.

[21] CD-adapco. User Guide. Star-CCM+ Version 9.04; 2014.

[22] Lee S-K, Chien H-P, Gu H. CFD Study of Deep Draft SemiSubmersible VIM. Offshore Technology Conference-Asia: Offshore Technology Conference; 2014.

[23] Tan JHC, Magee A, Kim JW, Teng YJ, Zukni NA. CFD Simulation for Vortex Induced Motions of a Multi-Column Floating Platform. ASME 2013 32nd International Conference on Ocean, Offshore and Arctic Engineering: American Society of Mechanical Engineers; 2013. p. V007T08A66-VT08A66.

[24] Koop A, Rijken O, Vaz G, Maximiano A, Rosetti G. CFD Investigation on Scale and Damping Effects for Vortex Induced Motions of a Semi-Submersible Floater. Offshore Technology Conference: Offshore Technology Conference; 2016.

[25] Blazek J. Computational fluid dynamics: principles and applications: Butterworth-Heinemann; 2015.

[26] Liang Y, Tao L. Interaction of vortex shedding processes on flow over a deep-draft semisubmersible. Ocean Engineering. 2017;141:427-49.

[27] Liang Y, Tao L, Xiao L, Liu M. Experimental and numerical study on vortex-induced motions of a deep-draft semi-submersible. Applied Ocean Research. 2017;67:169-87.

[28] Schewe G. On the force fluctuations acting on a circular cylinder in crossflow from subcritical up to transcritical Reynolds numbers. Journal of Fluid Mechanics. 1983;133:265-85. 

wake flow of a circular cylinder. Experiments in Fluids. 2010;48:1059-79.

724 [30] Hunt JC, Wray AA, Moin P. Eddies, streams, and convergence zones in turbulent flows. 1988.

725

[31] Liu M, Xiao L, Liang Y, Tao L. Experimental and numerical studies of the pontoon effect on vortex-induced motions of deep-draft semi-submersibles. Journal of Fluids and Structures. 2017;72:59-79.

727 


\section{List of Figures}

Fig. 1 Model configurations: (A) Four columns configuration (model I); (B) Four columns with pontoons connected configuration (model II)......

Fig. 2 Columns arrangement and flow direction.........................................................................6

Fig. 3 Experimental set-up in the circulating water channel.

Fig. 4 Visualization of the mesh (Case: FCP_N5) at the middle draft level of the four columns configuration model ( $X Y$ plane). Sub-picture (A) is a local zooming to show the "Prims Layer Mesher”. .7

Fig. 5 Convergence of mean drag force coefficient $(C D)$ for the four columns configuration. 10

Fig. 6 Convergence of root mean square lift force coefficient (CLrms) for the four columns configuration.

Fig. 7 Convergence of Strouhal number (St) for the four columns configuration.

Fig. 8 Convergence of mean drag force coefficient $(C D)$ for the four columns with pontoons connected configuration

Fig. 9 Convergence of root mean square lift force coefficient (CLrms) for the four columns with pontoons connected configuration.

Fig. 10 Convergence of Strouhal number $(S t)$ for the four columns with pontoons connected configuration.

Fig. 11 Time-averaged velocity distribution behind column 3 at the middle draft level of the four columns configuration in $X Y$ plane.

Fig. 12 Time-averaged velocity distribution behind column 3 at the middle draft level of the four columns with pontoons connected configuration in $X Y$ plane.

Fig. 13 Time-averaged flow properties of the Umean/UC in $X Y$ plane (middle draft level of the structure) for column 3, at $x / D=0.75 R e=4.3 \times 104$, "FC" is the four columns configuration and "FCP" is the four columns with pontoons connected configuration. 15

Fig. 14 Time-averaged flow properties of the $U i / U C$ (velocity component $i$ ) in $X Y$ plane (middle draft level of the structure) for column 3 at $x / D=0.75, R e=4.3 \times 104$. "FC" is the four columns configuration and "FCP" is the four columns with pontoons connected configuration. 

plane (middle draft level of the structure) for each column at $x / D=0.75, R e=4.3 \times 104$. "FC" is the four columns configuration and "FCP" is the four columns with pontoons connected configuration. 17

Fig. 16 Instantaneous flow fields around column 3 for the four columns configuration at $X Y$ plane

Fig. 17 Instantaneous flow fields around column 3 for the four columns with pontoons connected configuration at $X Y$ plane (middle draft level of the structure), where $\omega z D / U$ is the non-dimensional spanwise vorticity

Fig. 18 A time series of the non-dimensional spanwise vorticity $(\omega z D / U)$ contours around the four columns configuration at the middle draft level showing the instantaneous flow fields in $X Y$ plane at $R e=4.3 \times 104$

Fig. 19 A time series of the non-dimensional spanwise vorticity $(\omega z D / U)$ contours around the four columns with pontoons connected configuration at the middle draft level showing the instantaneous flow fields in $X Y$ plane at $R e=4.3 \times 104$.

Fig. 20 The streamlines along with the vorticity $(\omega z D / U)$ contours around column 4 (the four columns configuration) at different draft levels (a: middle draft level; b: $z=0.13075 \mathrm{~m}$ draft level; c: pontoon upper

Fig. 21 The streamlines along with the vorticity $(\omega z D / U)$ contours around column 4 (the four columns with pontoons connected configuration) at different draft levels (a: middle draft level; b: $z=0.13075 \mathrm{~m}$ draft level; c: pontoon upper face draft level) showing the flow separation in $X Y$ plane at $R e=4.3 \times 104$.

Fig. 22 The streamlines along with the vorticity $(\omega z D / U)$ contours around column 3 (the four columns configuration) at different draft levels (a: middle draft level; b: $z=0.13075 \mathrm{~m}$ draft level) showing the flow separation in $X Y$ plane at $R e=4.3 \times 104$.

Fig. 23 The streamlines along with the vorticity $(\omega z D / U)$ contours around column 3 (the four columns with pontoons connected configuration) at different draft levels (a: middle draft level; b: $z=0.13075 \mathrm{~m}$ draft level) showing the flow separation in $X Y$ plane at $R e=4.3 \times 104$.

Fig. 24. Mean pressure coefficient distributions of each column at middle draft level $(X / D=0$ is the 

with downstream distances $(x / D=0.25, x / D=0.75$ and $x / D=1.5$ ) at $y / D=0$ (the central line of column), $R e=4.3 \times 104$. "FC" is the four columns configuration and "FCP" is the four columns with pontoons connected configuration. " $z / D=0$ " is the bottom of the column and " $z / D=0.36$ " is the pontoon upper face level.

Fig. 26 Time-averaged flow properties of the $U k / U C$ (velocity component $k$ ) in $X Z$ plane for column 2 with downstream distances $(x / D=0.25, x / D=0.75$ and $x / D=1.5)$ at $y / D=0$ (the central line of column), $R e=4.3 \times 104$. "FC" is the four columns configuration and "FCP" is the four columns with pontoons connected configuration. " $\mathrm{z} / \mathrm{D}=0$ " is the bottom of the column and " $\mathrm{z} / \mathrm{D}=0.36$ " is the pontoon upper face level.

Fig. 27 Time-averaged flow properties of the $U k / U C$ (velocity component $k$ ) in $X Z$ plane for column 3 with downstream distances $(x / D=0.25, x / D=0.75$ and $x / D=1.5)$ at $y / D=0$ (the central line of column), $R e=4.3 \times 104$. "FC" is the four columns configuration and "FCP" is the four columns with pontoons connected configuration. " $z / D=0$ " is the bottom of the column and " $z / D=0.36$ " is the pontoon upper face level.

Fig. 28 Time-averaged flow properties of the Ui/UC (velocity component $i$ ) in $Y Z$ plane for column 1 with downstream distance at $x / D=0.75, R e=4.3 \times 104$. (a) is the four columns configuration and (b) is the four columns with pontoons.

Fig. 29 Time-averaged flow properties of the Ui/UC (velocity component $i$ ) in YZ plane for column 2 and 4 with downstream distance at $x / D=0.75, R e=4.3 \times 104$. (a) is the four columns configuration and (b) is the four columns with pontoons.

Fig. 30 Time-averaged flow properties of the Ui/UC (velocity component $i$ ) in YZ plane for column 3 with downstream distance at $x / D=0.75, R e=4.3 \times 104$. (a) is the four columns configuration and (b) is the four columns with pontoons.

Fig. 31 A time series of the non-dimensional transverse vorticity $(\omega y D / U)$ contours around the four columns configuration at the central line of column 1 and 3 showing the instantaneous flow fields in $X Z$ 
812 columns configuration at the central line of column 2 showing the instantaneous flow fields in $X Z$ plane at

814 Fig. 33 A time series of the non-dimensional transverse vorticity $(\omega y D / U)$ contours around the four columns with pontoons connected configuration at the central line of column 1 and 3 showing the instantaneous flow fields in $X Z$ plane at $R e=4.3 \times 104$.

Fig. $34 \mathrm{~A}$ time series of the non-dimensional transverse vorticity $(\omega y \mathrm{D} / \mathrm{U})$ contours around the four columns with pontoons connected configuration at the central line of column 2 showing the instantaneous flow fields in $X Z$ plane at $R e=4.3 \times 104$.

Fig. 35 A time series of the non-dimensional spanwise vorticity $(\omega z D / U=1.5)$ isosurfaces around the four columns configuration showing the instantaneous flow fields at $R e=4.3 \times 104$. The red line is the average outside line within one vortex shedding period showing the linear outer contour line of the isosurfaces.

Fig. 36 A time series of the non-dimensional spanwise vorticity $(\omega z D / U=1.5)$ isosurfaces around the four columns with pontoons connected configuration showing the instantaneous flow fields at $R e=4.3 \times 104$. The red line is the average outside line within one vortex shedding period showing the linear outer contour line of the isosurfaces.

Fig. 37 A time series of isometric view representation of $Q$-criterion of the four columns configuration

Fig. 38 A time series of isometric view representation of $Q$-criterion of the four columns with pontoons

Fig. 39 Mean drag coefficients $(C D)$ on each member of the models (FC represents the four columns

Fig. 40 Root mean square lift coefficients (CLrms) on each member of the models (FC represents the four columns configuration; FCP represents the four columns with pontoons connected configuration)..44

Fig. 41 Mean lift coefficient $(C L)$ on each member of the models (FC represents the four columns 


\section{List of Tables}

840 Table 1 Main characteristics of the four columns configuration (model I)

841 Table 2 Main characteristics of the four columns with pontoons connected configuration (model II). 4

844 Table 5 The chronological order of vortices genesis for each column

Table 6 The resulting non-dimensional coefficients CD, CLrms and St for the four columns

Table 7 The resulting non-dimensional coefficients CD, CLrms and St for the four columns with pontoons connected configuration (“num.” represents “numerical”; “exp.” represents “experimental”). .42 